\title{
An Evaluation of Atmospheric Pressure Ionization Techniques for the Analysis of $N$-Methyl Carbamate Pesticides by Liquid Chromatography Mass Spectrometry
}

\author{
Stephen Pleasance* \\ Institute for Marine Biosciemres, National Research Council of Canada, Halifax, Nova Scotia, Canada
}

Joseph F. Anacleto

Chemistry Department, Dalhousie University, Halifax, Nova Scotia, Canada

M. Ruth Bailey and David H. North

Health Protection Branch, Health and Welfare Canada, Halifax, Nova Scotia, Canada

\begin{abstract}
Atmospheric pressure ionization (API) techniques are evaluated for the mass spectral analysis of $\mathrm{N}$-methyl carbamate pesticides. Atmospheric pressure chemical ionization (APCI) using a heated nebulizer interface provided both protonated molecules and abundant, characteristic fragment ions. With ion spray (ISP; pneumatically assisted electrospray ionization), which utilizes a milder "ion evaporation" process, primarily protonated molecules were obtained, although fragment ions similar to those observed in APCI could be induced by variation of the API orifice voltage. Product ion spectra of ISP-derived protonated molecules, generated by tandem mass spectrometry using collision-induced dissociation, are also presented. The APCI and ISP spectra of the carbamates are compared to those obtained with a thermospray interface and also to their electron ionization and methane CI spectra obtained with a particle beam interface. For all four interfaces, combined liquid chromatography mass spectrometry methods using conventional (4.6 mm i.d.) columns are described for the separation and detection of pesticide mixtures. These methods are applied to the confirmatory analysis of three representative carbamate pesticides, spiked at the 0.1-ppm level in green peppers. For those carbamates amenable to gas chromatography mass spectrometry, comparative results are presented. (J Am Soc Mass Spectrom 1992, 3, 378-397)
\end{abstract}

$\mathrm{T}$ The increasing use of $\mathrm{N}$-methyl carbamate insecticides in agriculture demands the development of highly sensitive and selective analytical procedures to determine trace level residues of these compounds in crops and other food products [1]. Unfortunately, confirmatory methods for this class of pesticide are rather limited. The thermal lability of many pesticides prevents their direct analysis by gas chromatography (GC), although developments in capillary column supports have led to a few GC-based methods for specific carbamates [2, 3]. Other workers have employed derivatization techniques to overcome the problem of thermal lability [4]. High performance

\footnotetext{
*Under contract from SCIEX, 55 Glen Cameron Road, Thornhill, Ontario, Canada.

Address reprint requests to Stephen Pleasance, Institute for Marine Biosciences, National Research Council of Canada, 1411 Oxford Street. Halifax, Nova Scotia B3H 3Z1, Canada.
}

liquid chromatography (HPLC) is the technique most widely used to circumwent heat sensitivity for pesticide analysis, although this approach has been frustrated by the lack of a sensitive, selective detector. Ultraviolet [5] and electrochemical [6] detection have been used, and although both provide adequate sensitivity, they do not provide the specificity necessary for the analysis of moderately complex sample matrices. HPLC using postcolumn hydrolysis and derivatization with fluorescence detection (FLD) has been developed [7] and refined [8], and is currently the most widely used technique for the determination of carbamates in water [9] and in fruit and vegetables $[10,11]$. This technique relies on the postcolumn hydrolysis of the carbamate moiety to methylamine with subsequent derivatization to a fluorescent isoindole product.

The application of mass spectrometry to the regula- 
tory analysis of pesticides and industrial chemicals in food and feed commodities has been reviewed [12]. Because of the potential of combined liquid chromatography mass spectrometry (LC/MS) as the ideal confirmatory method for pesticides, its application to these compounds has followed closely the rapid development of the technique itself. Early in this development, both a moving-belt interface using chemical ionization (CI) [13, 14], and a direct liquid introduction (DLI) interface [15], were evaluated for the analysis of carbamates by LC/MS. Although both studies elegantly demonstrated the feasibility of LC/MS for multiresidue pesticide analysis, these particular techniques have been hampered either by lack of sensitivity or by thermal degradation of the sample.

The advent of thermospray (TSP) ionization provided the first robust interface for routine LC/MS applications. Using either conventional postcolumn addition of ammonium acetate, or flament- or discharge-assisted ionization, the technique has been applied to a wide range of compounds including carbamates and other pesticides $[16,17]$. Both TSP and the more recently introduccd particle beam (PB) LC/MS interfaces have been evaluated recently by the U.S. Environmental Protection Agency for the determination of nonvolatile organic compounds, including several carbamates, in aqueous environmental samples $[18,19]$. While the authors reported both techniques to have detection limits within the range of interest for environmental pollutants in wastewater $(\sim 1-25$ $\mu \mathrm{g} / \mathrm{L}$ ), they placed much emphasis on the lack of structurally significant fragment ions in TSP spectra compared with the ability of the PB interface, to provide conventional electron ionization (EI) mass spectra consistent with those from library data bases. They also stressed the inability of TSP to provide sufficiently stable ion currents over extended periods for quantitative analyses. A more recent investigation of TSP for carbamate standards, by using a triple quadrupole instrument, demonstrated that the necessary structural information could be provided by collisionally activated product ion spectra obtained with tandem mass spectrometry (MS/MS), although no LC/MS/MS results were presented [20]. The authors did, however, reiterate the problem of fluctuating ion signals with TSP.

As an alternative to HPLC, the low critical temperatures of the mobile phases used in supercritical fluid chromatography (SFC) allows separation under mild conditions favorable to thermally labile compounds. In addition, SFC can provide enhanced chromatographic efficiency relative to LC, and also the capability for separations of less volatile and higher molecular weight compounds than does GC. Both capillary column and packed column SFC have been coupled with mass spectrometry, and both types of SFC/MS have been applied to the analysis of carbamate standards [21, 22]. Although good sensitivity was re- ported for capillary SFC/MS using CI, to date neither technique has been applied to the analysis of real world samples.

Much of the recent interest in atmospheric pressure ionization mass spectrometry (API/MS) has been primarily due to the ability of liquid-phase "ion evaporation" ionization processes to produce multiply charged ions of high molecular weight biopolymers [23]. However, API is also proving to be an extremely efficient means of coupling chromatographic methods such as HPI.C, SFC, and capillary electrophoresis to the mass spectrometer. An excellent review has recently detailed the use of API/MS as a detection system for the separation sciences [24]. Ion spray (ISP) is a recently developed API technique [25] that is proving to be well suited to the LC/MS analysis of trace levels of polar and thermally labile compounds $[24,26]$. In contrast to the related technique of electrospray ionization (ESI) [23], nebulization plays an important role in the ISP process, permitting the use of higher flow rates of highly aqueous mobile phases [25]. In a complementary role to that of ISP, gas-phase atmospheric pressure chemical ionization (APCI) using a corona discharge is currently undergoing a renaissance owing to the introduction of a heated nebulizer (HN) interface for LC/MS and SFC/MS [24, 27].

This paper presents an evaluation of both ISP and APCI for the analysis of $N$-methyl carbamate pesticides, and compares their performance with results obtained using both PB and TSP interfaces. Combined LC/MS methods using conventional (4.6 $\mathrm{mm}$ i.d.) columns are described for the separation and detection of pesticide mixtures using all four interfaces. These methodologies are applied to the determination of three representative carbamate pesticides, spiked at the 0.1-ppm level in green peppers.

\section{Experimental}

\section{Materials}

All of the carbamate standards listed in Table 1 were obtained from the Health Protection Branch repository in Ottawa and used to prepare stock solutions of $0.5 \mathrm{mg} / \mathrm{mL}$ in methanol or acetone for LC/MS or GC/MS, respectively. Working solutions were prepared by mixing or diluting the stock solutions in the appropriate solvent. Omnisolve methanol was obtained from BDH Chemicals (Poole, UK). Reagent grade formic acid was obtained from Fisher Scientific (Fairlawn, NJ). GC grade acetone and dichloromethane were obtained from Burdick and Jackson (Muskegon, MI), and GC grade hexane was obtained from J. T. Baker Chemical (Phillipsburg, NJ). Aqueous solutions were prepared by using glass-distilled water further purified by passage through a Millipore Corp. (Bedford, MA) Mill-Q purification system. 
Table 1. Names, abbreviations, and structures of the eight carbamate pestcides examined

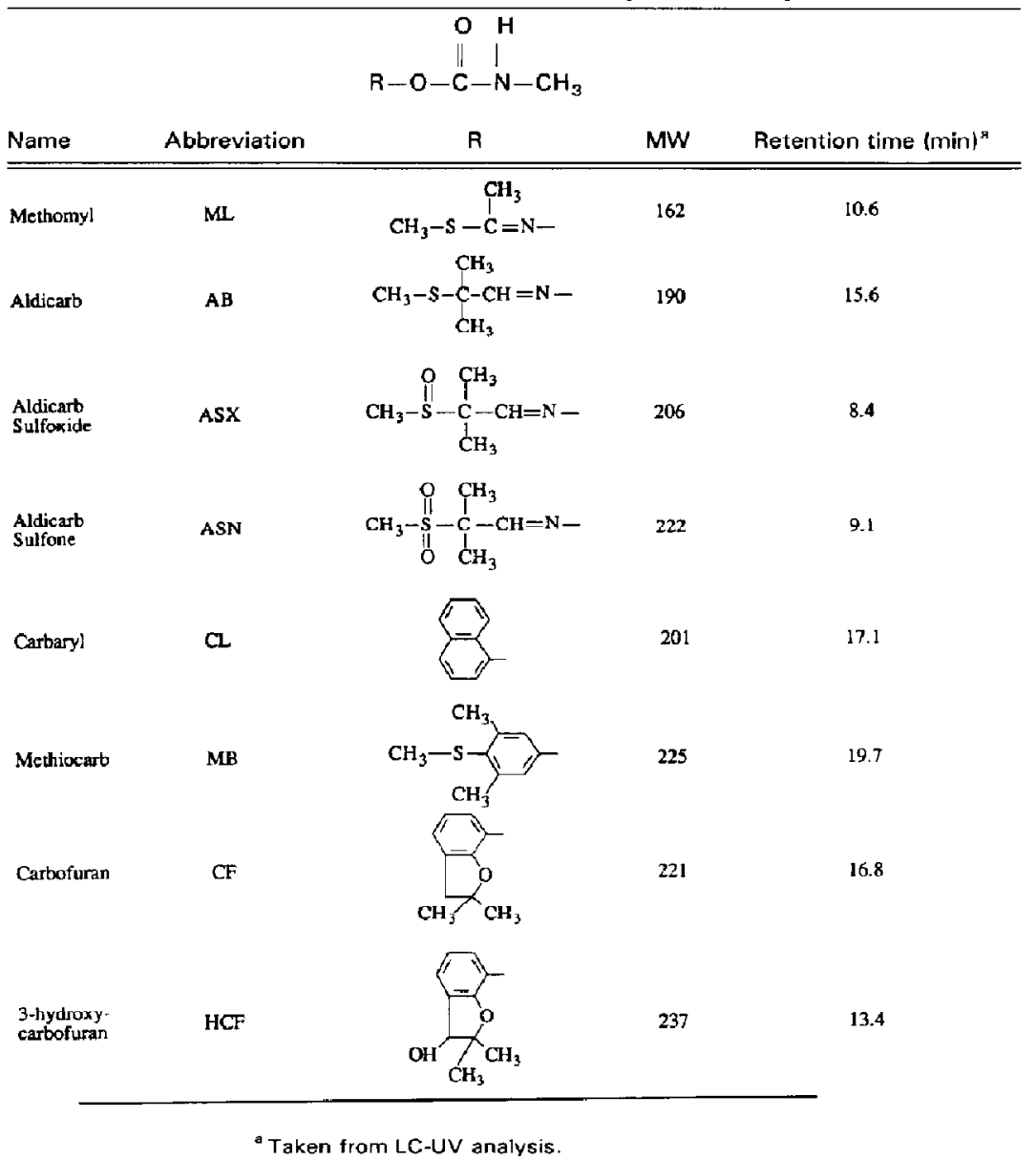

\section{Extraction}

The extraction procedures used in this investigation are based on those described previously [28]. In duplicate, macerated green peppers ( $35 \mathrm{~g}$ ) were mixed with acetone $(100 \mathrm{~mL})$ and homogenized in a Polytron homogenizer (Brinkmann Instruments Ltd., Rexdale, ON) for $1 \mathrm{~min}$. One of the homogenates was spiked with $7 \mathrm{~mL}$ of a solution containing $3.5 \mu \mathrm{g}$ each of methomyl, aldicarb and carbaryl in acetone $(0.1 \mathrm{ppm})$. The homogenates were treated separately in the laboratory and every effort made to prevent cross contamination. After centrifugation (2000 rpm for $5 \mathrm{~min}$ ), the supernatant was filtered through glasswool into a $500-m L$ separatory furmel. The residual pellet was homogenized for a further $30 \mathrm{~s}$ with dichloromethane $(100 \mathrm{~mL})$, and again centrifuged and filtered. Hexane $(100 \mathrm{~mL}$ ) was used to rinse the centrifuge buttle and filtered. The combined filtrate was shaken for $2 \mathrm{~min}$ and the lower aqueous layer was drained into a sepa- ratory funnel containing sodium chloride $(10 \mathrm{~g})$. The organic phase was filtered through sodium sulfate $(\sim 40 \mathrm{~g})$ on glasswool, into a round-bottomed flask. The aqueous phase was partitioned twice $(1 \mathrm{~min}$ of shaking) with dichloromethane $(2 \times 70 \mathrm{~mL})$, and the organic phase was filtered through sodium sulfate into the round-bottomed flask. The combined organic phase was evaporated to near dryness, made up to 20 $\mathrm{mL}$ with dichloromethane/hexane (50:50), and centrifuged (2000 rpm for $5 \mathrm{~min}$ ).

After filtering through a Millex-HV filter unit (Millipore), the extract was loaded into two 5-mL sample loops of a model 1002A Autoprep GPC (Analytical BioChemistry Laboratories Inc., Columbia, MO) containing a preconditioned $18.7-\mathrm{cm}$ column of Bio-Beads S-X3 (ABC) ( $30 \mathrm{~g})$. For both loops, the column was eluted with dichloromethane/hexane (50:50) at 5 $\mathrm{mL} / \mathrm{min}$ and after $9 \min (45 \mathrm{~mL})$ the eluent was collected for a further $19 \mathrm{~min}(95 \mathrm{~mL})$. In each case, after removal of the solvent, the residue was taken to 
near dryness with acetone or methanol $(\sim 10 \mathrm{~mL})$ and finally made up to $5 \mathrm{~mL}$ with either acetone or aqueous methanol (3:2) ready for GC/MS and LC/MS, respectively. A portion of the extract for LC/MS (1 $\mathrm{mL})$ was filtered with a syringe-tip filter prior to injection.

\section{GC/MS}

Analyses were performed on a HP5890A gas chromatograph coupled to a HP5988A quadrupole mass spectrometer (Hewlett-Packard Co., Palo Alto, CA). Manual cold needle, on-column injections $(1 \mu \mathrm{L})$ were made onto a J \& W Scientific (Rancho Cordova, CA) DB-17 fused silica capillary column $(30 \times 0.32 \mathrm{~mm}$ i.d.; $0.25-\mu \mathrm{m}$ phase thickness) using helium as the carrier gas. After an initial hold at $50^{\circ} \mathrm{C}$ for $1 \mathrm{~min}$, the oven was temperature programmed to $190^{\circ} \mathrm{C}$ at 20 ${ }^{\circ} \mathrm{C} / \mathrm{min}$ and then to $255^{\circ} \mathrm{C}$ at $8{ }^{\circ} \mathrm{C} / \mathrm{min}$. Positive ion EI spectra were obtained at $70 \mathrm{eV}$ and a source temperature of $150{ }^{\circ} \mathrm{C}$. Full-scan spectra were acquired from 50 to $250 \mathrm{u}$ at $1.16 \mathrm{scan} / \mathrm{s}$. For selected ion monitoring experiments a dwell time of $50 \mathrm{~ms} / \mathrm{u}$ was used.

\section{$P B$ LC/MS}

Analyses were performed on a Hewlett-Packard instrument consisting of a model 1090 Series II liquid chromatograph with a ternary PV5 solvent delivery system, a model 59980A PB interface, and a model 5988A quadrupole mass spectrometer. Flow injection analysis (FIA) was accomplished using a postinjector switching valve to bypass the column. Separations were achieved on a $4.6-\mathrm{mm}$ i.d. $\times 25-\mathrm{cm}$ column packed with $5 \mu \mathrm{m}$ Zorbax $\mathbf{R}_{X}$-C8 stationary phase. The column was protected by a guard column of the same phase. Aqueous methanol was used as the mobile phase at a flow rate of $1 \mathrm{~mL} / \mathrm{min}$. After an initial hold at 20\% methanol for $4 \mathrm{~min}$, a linear gradient to $70 \%$ methanol in 11 min was used, followed by a hold of $5 \mathrm{~min}$. Additional experiments were conducted with $0.01 \%$ ammonium acetate added to the water. An injection volume of $20 \mu \mathrm{L}$ was used and separations were performed at $40^{\circ} \mathrm{C}$. The column effluent was split (3:2) using a zero dead volume $T$ connector, with $\sim 0.4 \mathrm{~mL} / \mathrm{min}$ being fed to the PB interface. The split ratio was determined by the length of 0.005 -in i.d. stainless steel HPLC tubing on the waste side of the T. Ultrahigh purity helium (Liquid Carbonic, Scarborough, ON, Canada) was used as the nebulizing gas at a flow rate of $-1.5 \mathrm{~L} / \mathrm{min}$. The temperature of the desolvation chamber was maintained at $55^{\circ} \mathrm{C}$. Positive ion EI spectra were obtained at $70 \mathrm{eV}$ and a source temperature of $250{ }^{\circ} \mathrm{C}$. For $\mathrm{PB} / \mathrm{CI}$, methane was introduced into the source to give a pressure of $\sim 1.0 \times 10^{-4}$ torr, and the source temperature re- duced to $120^{\circ} \mathrm{C}$. Full-scan spectra were acquired from 50 to $250 \mathrm{u}$ at $0.54 \mathrm{scan} / \mathrm{s}$ or from 70 to $250 \mathrm{u}$ at 0.71 $\mathrm{scan} / \mathrm{s}$ for $\mathrm{CI}$ or when ammonium acetate was present in the mobile phase. For selected ion monitoring experiments a dwell time of $200 \mathrm{~ms} / \mathrm{u}$ was used.

\section{TSP LC/MS}

Analyses were performed on a system identical to that used for PB LC/MS except that a HP5988A TSP interface was used. The LC system was coupled directly to the TSP probe which was maintained at a stem temperature of $107^{\circ} \mathrm{C}$ and a tip temperature of $170^{\circ} \mathrm{C}$ for all FIA work. LC conditions were identical to those used in the PB LC/MS investigation except that $0.5 \mathrm{M}$ ammonium acetate was added postcolumn (using a Waters Associates [Milford, MA] 6000A LC pump) at a rate of $0.2 \mathrm{~mL} / \mathrm{min}$, for a final concentration of $\sim 0.08 \mathrm{M}$ and a combined mobile phase flow rate of $1.2 \mathrm{~mL} / \mathrm{min}$. For gradient elution LC/MS, the probe (stem) temperature was held at $110^{\circ} \mathrm{C}$ for 4 min and then programmed to $102^{\circ} \mathrm{C}$ at $-0.7^{\circ} \mathrm{C} / \mathrm{min}$. Full-scan spectra were acquired from 120 to $260 \mathrm{u}$ at $1.53 \mathrm{scan} / \mathrm{s}$ in both the filament-off and flamentassisted modes. For the latter, a voltage of $950 \mathrm{~V}$ was used. The source temperature was maintained at $276^{\circ} \mathrm{C}$.

\section{API LC/MS /MS}

Both LC/MS and LC/MS/MS experiments were performed on a HP1090 Series II liquid chromatograph, with a binary DR5 solvent delivery system and HP1050 autosampler, coupled to a SCIEX (Thornhill, ON, Canada) API III triple quadrupole mass spectrometer equipped with an API source, via either an $\mathrm{HN}$ or IonSpray interface. Ultrapure nitrogen (Canadian Liquid Air, Ltd.) was used as the curtain gas in the API source at a flow rate of $0.8-1.6 \mathrm{~L} / \mathrm{min}$. LC conditions were identical to those used in the PB LC/MS investigation, except that for LC/ISP/MS the mobile phase contained $0.1 \%$ formic acid. For LC/APCI/MS the column was coupled directly to the heated nebulizer interface, maintained at a temperature of $300-350{ }^{\circ} \mathrm{C}$, via a low dead volume injector (Valco CI4W; $1.0 \mu \mathrm{L}$ loop). The postcolumn injector was used to optimize the particular LC/MS system and for all FLA experiments. For the ISP interface, the column effluent was split using a zero dead volume $T$ connector, with a split ratio of 20:1 determined by the length of a fused silica capillary (50 $\mu \mathrm{m}$ i.d.) on the waste side of the $T$. The remainder of the column effluent was fed to the transfer line of the interface again via the postcolumn injector. The APCI discharge electrode and the ISP needle were maintained at 2.0 and $5.6 \mathrm{kV}$, respectively. High purity air was used as the nebulizing gas in both interfaces at flow rates of 1.2 and $0.6 \mathrm{~L} / \mathrm{min}$, respectively. The API source was maintained at $40^{\circ} \mathrm{C}$, 
and an extractor fan was used to remove solvent vapor from the source housing.

A Macintosh Ilx computer was used for instrument control, data acquisition and data processing. A dwell time of $5 \mathrm{~ms} / \mathrm{u}$ was used for full-scan $(50-350 \mathrm{u})$ LC/MS analyses. For the determination of carbamates in pepper extracts and the generation of calibration curves, selected ion monitoring (SIM) was used with a dwell time of $200 \mathrm{~ms} / \mathrm{u}$. A dwell time of $100 \mathrm{~ms} / \mathrm{u}$ was used for the SIM LC/MS analyses of mixtures of the pesticide standards. Tandem mass spectrometry measurements were based on collision-induced dissociations (CID) within the rf-only quadrupole at a collision energy of $30 \mathrm{eV}$ (laboratory frame). Argon was used as the target gas at an indicated thickness of $3.5 \times 10^{14}$ molecules $\mathrm{cm}^{-2}$. For the confirmation of pesticides in pepper extracts, selected reaction monitoring (SRM) was used with a dwell time of $200 \mathrm{~ms}$. Both product ion and neutral loss modes were used in LC/MS/MS analyses of mixtures of the pesticide standards.

\section{LC with Ultraviolet (UV) Detection}

LC-UV analyses of the standard mixture of pesticides were obtained on the $4.6-\mathrm{mm}$ i.d. column with LC conditions identical to those used in LC/PB/MS analyses (no ammonium acetate in the mobile phase). Detection was at $214 \mathrm{~nm}$ using a HP1050 diode array detector (DAD) and a HP79994A data system.

\section{Results and Discussion}

\section{FIA}

Table 1 gives the names, abbreviations, and structures of the $N$-methyl carbamate pesticides examined in this investigation. Of these compounds, $A B$ and its sulfonated metabolites, ASN and ASX, are bona fide LC/MS candidates that cannot be analyzed by GC/MS using routinely employed techniques (see ref 3 ) because of problems of thermal decomposition and/or volatility. In addition, ML is a highly probable LC/MS candidate owing to its poor GC retention characteristics. Initially, all of the carbamates were analyzed by FLA using the four interfaces to optimize mass spectrometry conditions for combined LC/MS.

ISP / MS and ISP/MS / MS. Figure 1a shows the fullscan, background subtracted ISP spectrum of ML acquired by FIA. The spectrum was obtained by the injection $(1 \mu \mathrm{L})$ of a standard solution $(0.25 \mathrm{mg} / \mathrm{mL})$ into a mobile phase of aqueous methanol (50:50) containing $0.1 \%$ formic acid at a flow rate of $50 \mu \mathrm{L} / \mathrm{min}$. The acid was included in the mobile phase to provide preformed ions in solution, thereby increasing the efficiency of the ion evaporation process. While the protonated molecule $\left(\mathrm{MH}^{+}\right.$ion) dominates the spec- trum at $m / z 163$, several fragment ions are observed at $m / z 122,106$, and 88 . During the optimization of the interface, it was noted that the intensities of these fragment ions could be increased by raising the voltage applied to the sampling orifice of the API source. This is illustrated in Figure $1 \mathrm{~b}$ which shows the spectrum of ML acquired at an orifice voltage of $70 \mathrm{~V}$. The base peak is no longer the $\mathrm{MH}^{+}$ion, but rather the fragment ion at $m / z 88$. In addition, several other fragment ions are now apparent. A similar but much less dramatic effect could be obtained by increasing the flow rate of nitrogen to the gas curtain in the API interface. Unfortunately, this increase in structural information (discussed below) was generally achieved at the expense of overall sensitivity as noted by the relative ion intensity scales of Figure $1 a$ and $b$. Conversely, any attempt to generate more abundant $\mathrm{MH}^{+}$ ions by further reduction in the orifice voltage was countered by a dramatic rise in chemical noise due to mobile phase cluster ions.

A compromise was reached, and the ISP mass spectra of the individual carbamates $(0.25 \mathrm{mg} / \mathrm{mL})$ were subsequently acquired by FIA using an orifice potential of $55 \mathrm{~V}$ and a curtain gas flow rate of 1.2 $\mathrm{L} / \mathrm{min}$. The major ions observed, and their relative intensities, are listed in Table 2. Under these conditions, the $\mathrm{MH}^{+}$ion was the base peak in the spectra of all of the carbamates except AB. This is in contrast to the TSP spectra of these compounds (see below), where all but two of the carbamates (ML and CF) were observed only as their ammonium adduct ions, $\left[\mathrm{M}+\mathrm{NH}_{4}\right]^{+}$. Also in contrast to the TSP spectra is the appearance of several characteristic, common fragment ions in the ISP spectra. All of the carbamates, except AB and its sulfoxide (ASX), showed a characteristic loss of methyl isocyanate $\left(\mathrm{CH}_{3} \mathrm{NCO}\right)$ to give an ion at [MH-57] ${ }^{+}$, as exemplified by the ion at $\mathrm{m} / \mathrm{z}$ 106 in the spectrum of ML (Figure 1). A similar loss has been reported in both the $\mathrm{CI}$ and EI spectra of these compounds $[29,30]$ which will also be discussed later. This fragmentation is particularly favored in the aromatic carbamates $\mathrm{CL}, \mathrm{MB}, \mathrm{CF}$, and $\mathrm{HCF}$, where charge retention is resonance stabilized after cleavage of the single carbon-oxygen bond, and is illustrated by the abundant ion at $\mathrm{m} / \mathrm{z} 145$ in the spectrum of CL (Table 2). For $A B$ and ASX (Figure 2a) the more favorable loss of carbamic acid $\left(\mathrm{CH}_{3} \mathrm{NHCO}_{2} \mathrm{H}\right)$, to give the corresponding protonated nitrile moiety, results in the characteristic $[\mathrm{MH}-75]^{+}$ions at $m / z 116$ and 132 , respectively. This ion is also observed at $m / z 88$ in the spectrum of ML (Figure 1). Other characteristic fragment ions observed for individual carbamates include: an ion at $m / z 220$ in the spectrum of HCF due to the elimination of water from the protonated molecule; an ion at $m / z 122$ in the spectrum of ML corresponding to the loss of acetonitrile (41 u); and an ion at $m / z 89$ in the spectrum of ASX (Figure 2), which is assigned to $\left[\mathrm{C}_{4} \mathrm{H}_{9} \mathrm{~S}\right]^{+}$, with the charge presumably stabilized by resonance between a 

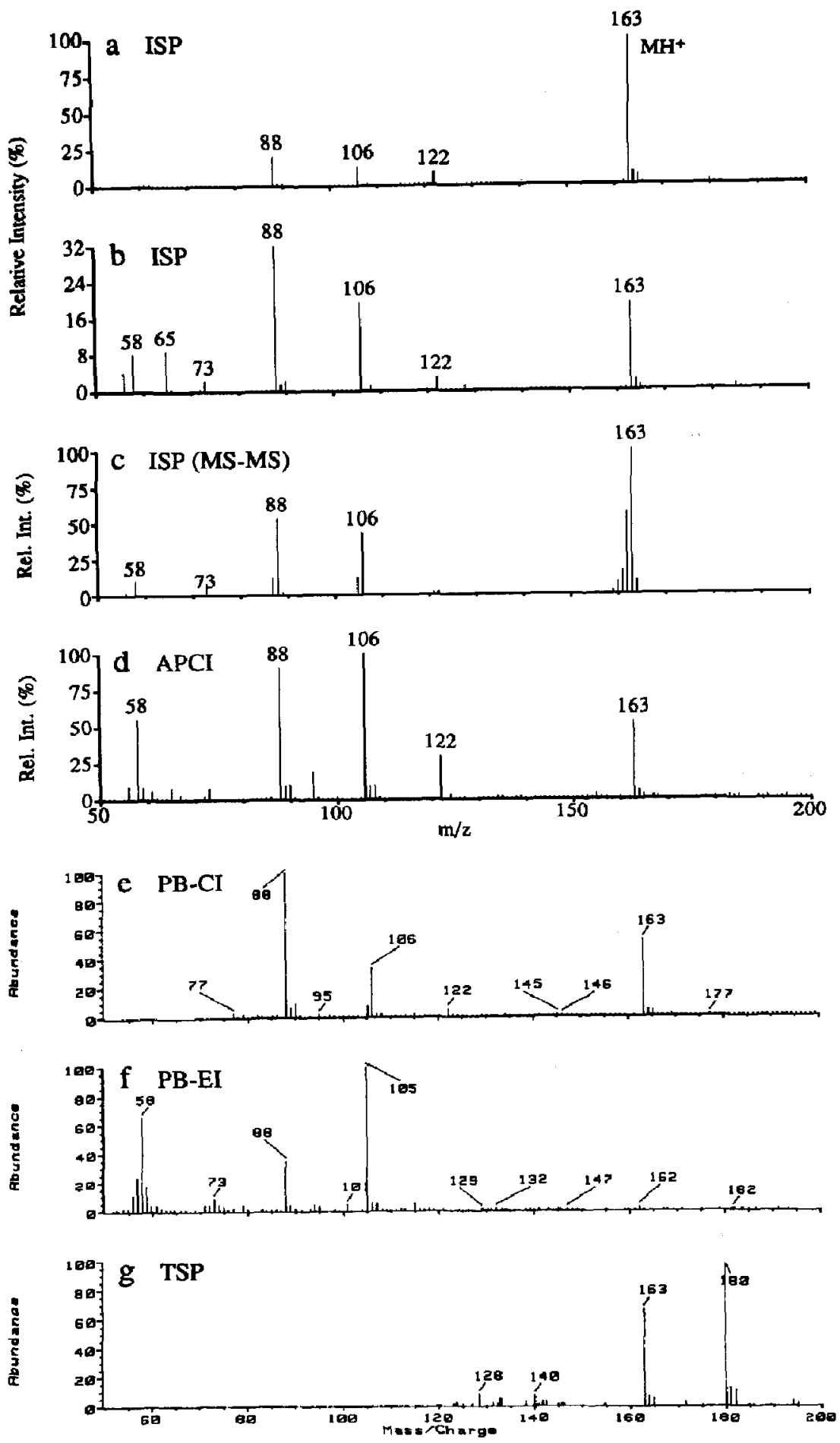

Figure 1. Background-subtracted FIA spectra of methomyl (ML) obtained by ISP/MS ( $250 \mathrm{ng})$ at orifice voltages of (a) $50 \mathrm{~V}$ and (b) 70 $\mathrm{V}$, (c) ISP/MS/MS (250 ng), (d) APCl-MS (50 ng), (e) PB-CI (2 $\mu \mathrm{g})$, (f) PB-EI $(2 \mu \mathrm{g})$, and (g) TSP/MS (250 ng). Conditions: mobile phase, aqueuds methanol for $d$, e, and $f$, modified with $0.1 \%$ formic acid for $a, b$, and $c$ and with 0.08 $\mathrm{M}$ ammonium acetate for $\mathrm{g}$. Flow rate, $50 \mu \mathrm{L} / \mathrm{min}$ for $\mathbf{a}, \mathbf{b}$, and $c$; $600 \mu \mathrm{L} / \mathrm{min}$ for d; $400 \mu \mathrm{L} / \mathrm{min}$ for $e$ and $f$; and $1.2 \mathrm{~mL} / \mathrm{min}$ for $g$. Injection volume, $1 \mu \mathrm{L}$ for $\mathbf{a}, \mathbf{b}, \mathbf{c}$, and $d$; and $5 \mu \mathrm{L}$ for $e, f$, and $g$. $A$ collision energy of $30 \mathrm{eV}$ and a target gas thickness of $3.5 \times 10^{14}$ molecules $\mathrm{cm}^{-2}$ was used for $\mathrm{c}$. 
tertiary carbonium ion and a sulferium structure with charge localized on the $S$ atom.

The above fragmentations were confirmed using the MS/MS capability of the triple quadrupole used for the API studies. In the product ion scan mode, the $\mathrm{MH}^{+}$ions selected by the first quadrupole $\left(Q_{1}\right)$ were introduced into the rf-only quadrupole $\left(Q_{2}\right)$ where they were made to undergo CID. The fragment ions thus generated were then mass analyzed by the third quadrupole $\left(\mathrm{Q}_{3}\right)$. The $30-\mathrm{eV}$ product ion spectrum of the $\mathrm{MH}^{+}$ion of $\mathrm{ML}$ is presented in Figure $1 \mathrm{c}$, and is very similar to the conventional ISP spectrum obtained with the orifice at $70 \mathrm{~V}$ (Figure $1 \mathrm{~b}$ ). The ion at $\mathrm{m} / \mathrm{z} 58$ is assigned to the protonated methyl isocyanate moiety, while the ion at $\mathrm{m} / z 73$ is rationalized as $\left[\mathrm{C}_{3} \mathrm{H}_{5} \mathrm{~S}\right]^{+}$resulting from protonation at the tertiary nitrogen, followed by hydrogen abstraction and loss of a carbamoylamine moiety.

The major ions observed in the product ion spectra of the $\mathrm{MH}^{+}$ions of the other carbamates obtained by FIA are listed in Table 2. The spectra for $\mathrm{ML}, \mathrm{CI}$, and CF are similar to the CID spectra of the $\mathrm{MH}^{+}$ions generated by methane $\mathrm{CI}$ [31] and by TSP [20]. However, for AB, ASX, ASN, and HCF in which the more abundant $\left[\mathrm{M}+\mathrm{NH}_{4}\right]^{+}$ion was used as the precursor in the TSP MS/MS study, the CID spectra are signifcantly different from those recorded for the $\mathrm{MH}^{+}$ion with ISP. For example, the CID spectrum of the [M+ $\left.\mathrm{NH}_{4}\right]^{+}$ion of ASX obtained by TSP [20] consisted simply of an intense ion at $m / z 89\left(\left[\mathrm{C}_{4} \mathrm{H}_{9} \mathrm{~S}\right]^{+}\right)$together with an unassigned ion at $m / z 149$, and bears little resemblance to the informative CID spectrum of the $\mathrm{MH}^{+}$ion of ASX presented in Figure 2c. Although the authors of this study [20] indicated that they observed no major ammonium cationized fragment ions, the unassigned ion at $m / z 149$ is readily interpretable as an ammoniated version of the $[\mathrm{MH}$ $75]^{+}$ion which is clearly observed at $m / z 132$ in the ISP MS/MS spectrum (Figure 2c). In addition, an ion is observed at $m / z 105$ in Figure $2 c$, corresponding to the loss of $\mathrm{HCN}(27 \mathrm{u})$ from the $[\mathrm{MH}-75]^{+}$fragment ion, together with an ion at $m / z 86$, which is probably related to the $\left[\left(\mathrm{CH}_{3}\right)_{2} \mathrm{CCHNOH}\right]^{+}$fragment ion which has been observed previously in the $\mathrm{CI}$ spectra of related aldicarb homologues [30]. In general, more abundant fragment ions were observed in the CID spectra generated by ISP than those reported previously for precursor ions generated by TSP [20].

APCI/MS. After optimization of the $\mathrm{HN}$ interface, the APCI spectra of the individual carbamates were acquired by FIA under conditions identical to those used for ISP/MS except that the mobile phase was at a flow rate of $600 \mu \mathrm{L} / \mathrm{min}$ and contained no acid. The major ions and their intensities are again listed in Table 2, and the background-subtracted APCI spectra of ML and ASX (50 ng) are presented in Figures 1d and $2 \mathrm{~d}$, respectively. While the majority of the $\mathrm{MH}^{+}$ ions remain prominent (the base peak in the spectra of ASN, MB, and CF), more abundant fragment ions are observed in the APCI spectra. The base peaks in the spectra of ML (Figure 1d) and CL are now the [MH-57] $^{+}$ions at $m / z 106$ and 145 , respectively, while the $[\mathrm{MH}-75]^{+}$ion is the most abundant for $A B$, ASX (Figure 2d), and HCF. In comparing the different API spectra, it should be noted that the solutions used for ISP were diluted fivefold for APCI. There is a considerable degree of similarity between the APCI mass spectra of the carbamates and their respective product ion spectra obtained by ISP/MS/MS, as typified by the spectra of ML in Figure $2 \mathrm{~d}$ and c, respectively. This similarity arises from CID occurring within the sampling orifice/expansion region of the API source. This is inherent in this particular API design, since some CID is necessary to decluster solvated analyte ion clusters. Additional informative fragment ions were observed in the APCI spectra of several of the carbamates, such as the ion at $m / z 135$ in the spectrum of HCF which is assigned to the loss of $\mathrm{CO}$ $(28 \mathrm{u})$ from the abundant $[\mathrm{MH}-75]^{+}$ion at $m / z 163$. Despite the abundance of fragmentation in the APCI spectra, little evidence of thermal decomposition was observed when using the HN interface. Under the conditions used for FIA, the optimal temperature of the IIN interface for maximum analyte signal $\left(300^{\circ} \mathrm{C}\right)$ was almost the same for all of the carbamates examined (the actual temperature of the vapor exiting the HN probe is $\sim 150^{\circ} \mathrm{C}$ ). The major factors influencing this optimal temperature were the mobile phase flow rate and composition (see below), and also the nebulizer gas flow rate. The optimal temperature of the interface was found to decrease with increasing percentages of methanol in the mobile phase. This is a similar situation to that found for TSP, but is much less dramatic and compound dependent. The practical implications of these observations with regard to gradient elution LC/API/MS are discussed below.

It should be noted that all of the API spectra presented thus far have been background subtracted to eliminate abundant low mass ions $(<150 \mathrm{u}$ ), corresponding to cluster ions of the mobile phase. Declustering can be accomplished by increasing the orifice voltage and/or the curtain gas flow rate. However, as noted above, both strategies result in a decrease of the analyte signal. Although acetonitrile generally provides less background chemical noise than methanol in both ISP and APCl, the latter solvent was used in the present comparative study because it was found to be the optimal solvent for the analysis of these compounds by LC/PB/MS. The percentage of methanol in the mobile phase did not appear to signifcantly affect the quality of spectra obtained by either API technique. Under ISP conditions most carbamates gave a slightly improved response at lower percentages of methanol. This improvement was offset, however, by a general increase in background chemical noise with decreasing organic content in the mobile phase. 
jor ions observed by FIA using various LC/MS interfaces

\begin{tabular}{|c|c|c|c|c|c|c|}
\hline \multirow{2}{*}{$\begin{array}{l}\text { Ionization } \\
\text { techniques }\end{array}$} & \multicolumn{5}{|c|}{ Major ions and their intensity } & \multirow{2}{*}{$\begin{array}{r}\text { LC/MS m } \\
\text { detection lin } \\
\text { SIM }\end{array}$} \\
\hline & {$\left[\mathrm{M}+\mathrm{NH}_{4}\right]^{+}$} & {$\left[\mathrm{MH}^{+} / \mathrm{IMI}^{+1}\right.$} & {$[\mathrm{MH}-57]^{+} /[\mathrm{M}-57]^{+*}$} & [MH-75] $^{+}$ & Other ions & \\
\hline ISP/MS & & $163(100)^{b}$ & $106(12)$ & $88(25)$ & $122(8)$ & 0.40 \\
\hline ISP/MS/MS & & $163(100)$ & $106(45)^{b}$ & $88(52)$ & $122(1), 73(9), 58(10)$ & - \\
\hline APCI & & $163(53)$ & $106(100)^{b}$ & $88(90)$ & $122(23), 73(7), 58(54)$ & 0.06 \\
\hline $\mathrm{PB}-\mathrm{Cl}$ & & $163(68)^{b}$ & $106(28)$ & $88(100)$ & $145(2), 134(2), 122(7)$ & - \\
\hline PB-EI & & $162(3)$ & $105(100)^{b}$ & - & $88(30), 73(15), 58(70)$ & $254^{\circ}$ \\
\hline TSP & $180(100)^{\mathrm{b}}$ & $163(85)$ & & & & $2.8^{c}$ \\
\hline ISP/MS & & $191(55)^{b}$ & - & $116(100)$ & $89(13), 70(9)$ & 1.5 \\
\hline ISP/MS/MS & & $191(90)$ & - & $116(85)$ & $89(100)$ & - \\
\hline $\mathrm{APCl}$ & & $191(3)$ & $134(4)$ & $116(100)^{b}$ & $148(4), 89(59), 86(12), 70(52)$ & 0.07 \\
\hline $\mathrm{PC}-\mathrm{Cl}$ & & & & $116(45)^{b}$ & $145(2), 89(100)$ & - \\
\hline PB-El & & & & $115(80)^{b}$ & $89(100)^{b}, 58(100)$ & $>50$ \\
\hline TSP & $208(100)^{b}$ & &. & & & $0.9^{c}$ \\
\hline ISP/MS & & $207(100)$ & - & $132(33)$ & & $1.0^{\mathrm{c}}$ \\
\hline ISP/MS/MS & & $207(100)$ & - & $132(55)$ & $144(6), 105(10), 89(25), 86(40), 76(15), 69(10)$ & - \\
\hline APCI & & $207(22)$ & $150(12)$ & $132(100)^{\mathrm{b}}$ & $144(19), 105(13), 89(20), 86(44), 76(60), 69(21)$ & $0.18^{\prime}$ \\
\hline PB-Cl & & $207(8)$ & 150(9) & $132(100)^{b}$ & $144(7), 105(10), 97(8), 89(15), 86(42)$ & - \\
\hline PB-El & & & - & $131(8)$ & $143(15), 122(10), 86(100), 68(80), 64(90), 58(60)$ & $152^{\mathrm{c}}$ \\
\hline TSP & $224(100)^{\mathrm{b}}$ & & & & & $2.5^{\mathrm{c}}$ \\
\hline ISP/MS & & $223(100)$ & 166(5) & $148(1)$ & & $1.8^{\mathrm{c}}$ \\
\hline ISP/MS/MS & & $223(100)$ & $166(18)$ & $148(30)$ & $86(35), 76(20), 58(10)$ & - \\
\hline $\mathrm{APCl}$ & & $223(100)^{b}$ & $166(28)$ & $148(13)$ & $86(19), 76(22)$ & $0.1^{\mathrm{c}}$ \\
\hline PB-Cl & & $223(65)$ & $166(30)$ & $148(100)^{b}$ & $180(19), 150(25), 86(60), 81(40)$ & - \\
\hline PB-EI & & & - & - & $143(15), 122(5), 86(100)^{b}, 68(80), 58(35)$ & $79^{c}$ \\
\hline TSP & $240(100)^{b}$ & $224(2)$ & & & & $1.1^{\mathrm{c}}$ \\
\hline
\end{tabular}


jior ions observed by FIA using various LC/MS interfaces (Continued)

\begin{tabular}{|c|c|c|c|c|c|c|}
\hline \multirow{2}{*}{$\begin{array}{l}\text { lonization } \\
\text { techniques }\end{array}$} & \multicolumn{5}{|c|}{ Major ions and their intensity } & \multirow{2}{*}{$\begin{array}{r}\text { LC/MS } \\
\text { detection } \\
\text { SI }\end{array}$} \\
\hline & {$\left[\mathrm{M}+\mathrm{NH}_{4}\right]^{+}$} & $/[\mathrm{MH}]^{+} /\left[\mathrm{M}^{+*}\right.$ & {$[\mathrm{MH}-57]^{+} /[\mathrm{M}-57]^{+}$} & {$[\mathrm{MH}-75]^{+}$} & Other ions & \\
\hline ISP/MS & & $202(100)^{b}$ & $145(50)$ & - & & 1.0 \\
\hline ISP/MS/MS & & $202(55)$ & $145(100)$ & $127(25)$ & $117(18)$ & - \\
\hline $\mathrm{APCl}$ & & $202(47)$ & $145(100)^{\mathrm{b}}$ & - & $159(7)$ & 0.05 \\
\hline $\mathrm{PB}-\mathrm{Cl}$ & & $202(62)$ & $145(100)^{\mathrm{b}}$ & - & $173(12), 115(2)$ & - \\
\hline PB-EI & & $201(5)$ & $144(100)^{*}$ & - & $145(12), 116(45), 115(75), 89(12), 63(10)$ & 10 \\
\hline TSP & $219(100)^{b}$ & & & & & 0.8 \\
\hline ISP/MS & & $22611001^{6}$ & 169(32) & - & - & $0.6^{\circ}$ \\
\hline ISP/MS/MS & & $226(92)$ & $169(97)$ & - & $154(5), 93(4)$ & - \\
\hline $\mathrm{APCl}$ & & $226(100)^{\mathrm{D}}$ & $169(96)$ & - & $121(9)$ & 0.07 \\
\hline $\mathrm{PB}-\mathrm{Cl}$ & & $226(100)^{b}$ & $169(49)$ & - & $197(9), 145(2)$ & - \\
\hline PB-El & & $225(5)$ & $168(100)^{b}$ & - & $153(60), 109(40), 91(25), 77(15), 65(10)$ & $19^{\circ}$ \\
\hline TSP & $243(100)^{b}$ & $226(1)$ & & & & $0.8^{\circ}$ \\
\hline ISP/MS & & $222(100)^{b}$ & $165(24)$ & - & - & $0.3^{c}$ \\
\hline ISP/MS/MS & & $222(100)$ & $165(75)$ & $147(4)$ & $123(75), 137(6)$ & - \\
\hline $\mathrm{APCl}$ & & $222(100)^{\mathrm{b}}$ & $165(64)$ & - & $123(5)$ & 0.05 \\
\hline $\mathrm{PB}-\mathrm{Cl}$ & & $222(100)^{\mathrm{b}}$ & $165(50)$ & - & $193(11), 145(2), 123(3), 90(2)$ & - \\
\hline PB-EI & & $221(4)$ & $164(100)^{b}$ & $146(20)$ & $\begin{array}{l}149(90), 131(35), 123(35), 122(30), 103(20) \\
77(25), 65(20)\end{array}$ & $55^{c}$ \\
\hline TSP & $239(100)^{\mathrm{b}}$ & $222(25)$ & & & & $0.8^{\circ}$ \\
\hline ISP/MS & & $238(100)^{b}$ & $181(8)$ & $163(5)$ & $220(70)$ & $1.0^{\circ}$ \\
\hline ISP/MS/MS & & $238(100)$ & $181(60)$ & $163(92)$ & $220(46), 145(18), 135(26), 117(5)$ & - \\
\hline $\mathrm{APCl}$ & & $238(6)$ & - & $163(100)^{\mathrm{b}}$ & $220(52), 135(17), 107(5)$ & 0.07 \\
\hline $\mathrm{PB}-\mathrm{Cl}$ & & $238(50)$ & $181(55)$ & $163(35)$ & $\begin{array}{l}220(100)^{b}, 209(10), 191(5), 145(3), 137(4) \\
111(1)\end{array}$ & - \\
\hline PB-EI & & $237(3)$ & $180(50)$ & $162(20)$ & $\begin{array}{l}151(20), 147(30), 137(100)^{b}, 123(11), 91(13) \\
65(10)\end{array}$ & $7^{\mathrm{c}}$ \\
\hline TSP & $255(100)^{b}$ & & & & $222(2)$ & $1.1^{\mathrm{c}}$ \\
\hline
\end{tabular}

jected on-column for $S / N>3 .{ }^{\circ}$ Ions used for $S I M$ experiments and detection limit. "Estimated from LC/MS relative response under gradient elution. 

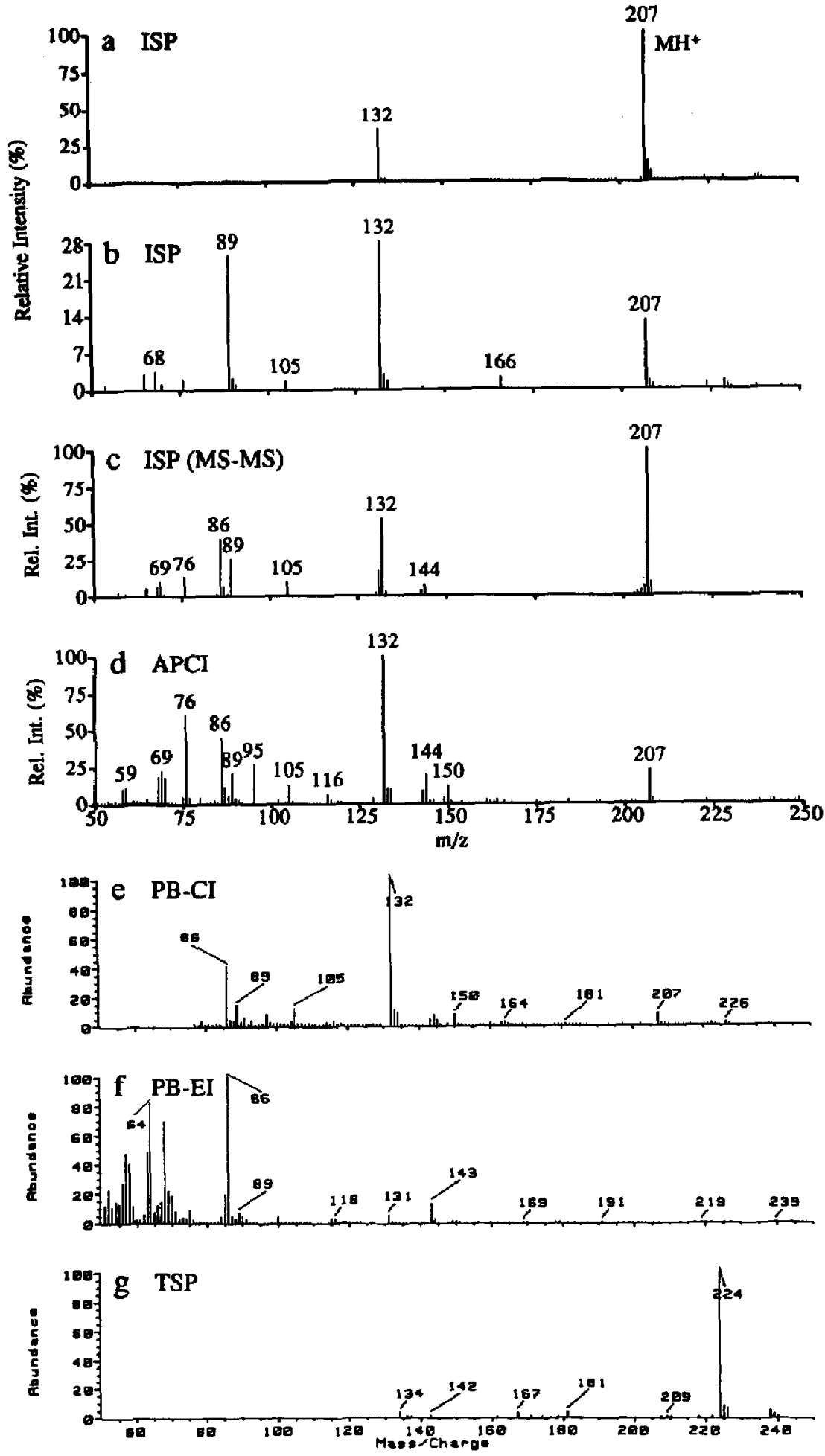

Figure 2. Background-subtracted spectra of aldiçarb sulfoxide (ASX) obtained by ISP/MS at orifice voltages of (a) $50 \mathrm{~V}$ and (b) $70 \mathrm{~V}$, (c) ISP/MS/MS, (d) APCl/MS, (e) PB-CI (f) PB-EI, and (g) TSP/MS. Conditions as in Figure 1. 
$P B / M S$. The full-scan EI and methane CI mass spectra of ML $(2 \mu \mathrm{g})$ obtained by FIA with the PB interface are shown in Figure 1, $f$ and $1 e$, respectively. Each of these was obtained using aqueous methanol (50:50) at a flow rate of $0.4 \mathrm{~mL} / \mathrm{min}$. The PB-EI spectrum of MT. compares favorably with that of the GC acquired spectrum from the Health Protection Branch library data base. Only a weak molecular ion at $m / z 162$ is observed, and the base peak is the $[\mathrm{M}-57]^{+}$radical cation at $m / z 105$ (loss of $\mathrm{CH}_{3} \mathrm{NCO}$ ). Under methane $\mathrm{CI}$ conditions (Figure 1e) the protonated molecule $(m / z 163)$ is more in evidence and the base peak becomes the $[\mathrm{MH}-57]^{+}$fragment at $m / z$ 106. The major ions observed for the individual carbamates under both $\mathrm{EI}$ and methane $\mathrm{CI}$ conditions with the $\mathrm{PB}$ interface are listed in Table 2. The EI spectrum of ASX is presented in Figure $2 f$. The molecular ion ( $m / z 206$ ) is not observed for this compound and its spectrum is almost identical to that obtained for the related ASN. The two analogues may be differentiated by their $\mathrm{CI}$ spectra, however, which provide both $\mathrm{MH}^{+}$and $[\mathrm{MH}-75]^{+}$ions. A comparison of Figure $2 \mathrm{~d}$ and $\mathrm{e}$ reveals a similarity between the $\mathrm{APCI}$ and $\mathrm{PB}-\mathrm{CI}$ spectra of $A S X$, and this was generally true of all the carbamates examined. Other than the relative intensities of the fragment ions, the only significant difference between the spectra was the appearance of ions corresponding to $\left[\mathrm{M}+\mathrm{C}_{2} \mathrm{H}_{5}-57\right]^{+}$in the $\mathrm{PB}-\mathrm{CI}$ spectra of the aromatic carbamates. This is illustrated by the ion at $m / z 197$ in the spectrum of MB.

It was immediately apparent that significantly larger amounts of the carbamates were required to obtain full-scan spectra of comparable quality by $\mathrm{PB}-\mathrm{EI}$ and $\mathrm{PB}-\mathrm{CI}$, than by APCI with the $\mathrm{HN}$ interface and indeed by ISP/MS/MS. The parent molecule of the sulfonated compounds, $\mathbf{A B}$, gave an extremely poor response under both $\mathrm{PB}-\mathrm{EI}$ and $\mathrm{PB}-\mathrm{CI}$ conditions; in our hands, no reproducible, library-searchable EI spectrum could be obtained with the $\mathrm{PB}$ interface, even for an injection of $2 \mu \mathrm{g}$. The response of ML was also notably weak. Improved responses were obtained for the majority of the carbamates at higher percentages of methanol, with no significant changes in the spectral quality. The relative sensitivities of the individual interfaces will be discussed in more detail later.

TSP/MS. The TSP spectrum of ML (250 $\mathrm{ng}$ ) obtained by FIA is shown in Figure 1g. This was obtained using a mobile phase of aqueous methanol (50:50) with the postinjector addition of $0.2 \mathrm{~mL} / \mathrm{min}$ of $0.5 \mathrm{M}$ ammonium acetate for a combined flow rate of $1.2 \mathrm{~mL} / \mathrm{min}$ and final concentration of ammonium acetate of $\sim 0.08 \mathrm{M}$. The protonated and ammoniated species at $m / z 163$ and 180 , respectively, dominate the spectrum with little evidence of fragmentation. Although the $\mathrm{MH}^{+}$ion is also much in evidence in the TSP spectrum of $\mathrm{CF}$, only the $\left[\mathrm{M}+\mathrm{NH}_{4}\right]^{+}$ions are observed for the remainder of the carbamates
(Table 2). Variation of the percentage of methanol in the mobile phase did affect the response of individual carbamates, but these changes could be compensated to some degree by appropriate changes in the vaporizer temperature. Unfortunately, the accurate control of the TSP operating temperatures has been identified as a requirement for ion stability in LC/MS [18], and this can cause problems during gradient elution (see below). All of the spectra were acquired with filament assisted ionization because this appeared to provide more stable ion currents than with conventional (filament-off) ionization. These observations are all in agreement with those reported previously for the carbamates $[16,18,20]$. The fact that the majority of the ion current in TSP and ISP is associated with one quasi-molecular species (i.e., $\left[\mathrm{M}+\mathrm{NH}_{4}\right]^{+}$or $\mathrm{MH}^{+}$ ion, respectively), suggests that these techniques should be ideal for SIM. Interestingly, at high sample concentrations, ions corresponding to the dimer [2M $+\mathrm{H}^{+}$of each carbamate were observed under both TSP and ISP conditions.

Although good sensitivity and linearity for standard solutions of carbamates were observed using FIA with ISP, TSP, and APCI using SIM techniques (data not shown), this method of sample introduction has several obvious drawbacks for the analysis of complex extracts containing salts and other endogenous material. The simultaneous introduction of many possible sources of interferences into the ion source with the analyte effectively prevents the use of FIA for quantitative analyses. Although the use of MS/MS techniques in conjunction with FLA can provide additional selectivity to filter out many potential interferences, there still remain the problems of isomeric compounds and other components influencing the ionization efficiency. Effective sample cleanup is necessary for quantitative work, and the easiest way to accomplish this without introducing additional sample handling steps is to provide a chromatographic separation prior to ionization.

\section{$L C / M S$}

Despite the poor UV response of the majority of the carbamates (with the exception of $\mathrm{CL}$, which contains a strongly absorbing naphthalene moiety), a simple gradient elution LC method was developed off-line using UV detection. The UV chromatogram (214 nm) showing the separation of the eight carbamate pesticides $(20 \mu \mathrm{g} / \mathrm{mL})$, on a $4.6-\mathrm{mm}$ i.d. base-deactivated $\mathrm{C}_{\mathrm{g}}$ column using a mobile phase of aqueous methanol, is presented in Figure 3a. The carbamates are adequately resolved for the purposes of this investigation and the individual retention times are given in Table 1. Slight differences in retention times were observed between the traces in Figure $3 a, d$, and $e$, and those in Figure $3 b$ and $c$ (particularly noticeable for the early eluting ASX and ASN). These differences were attributable to the different solvent delivery systems of 

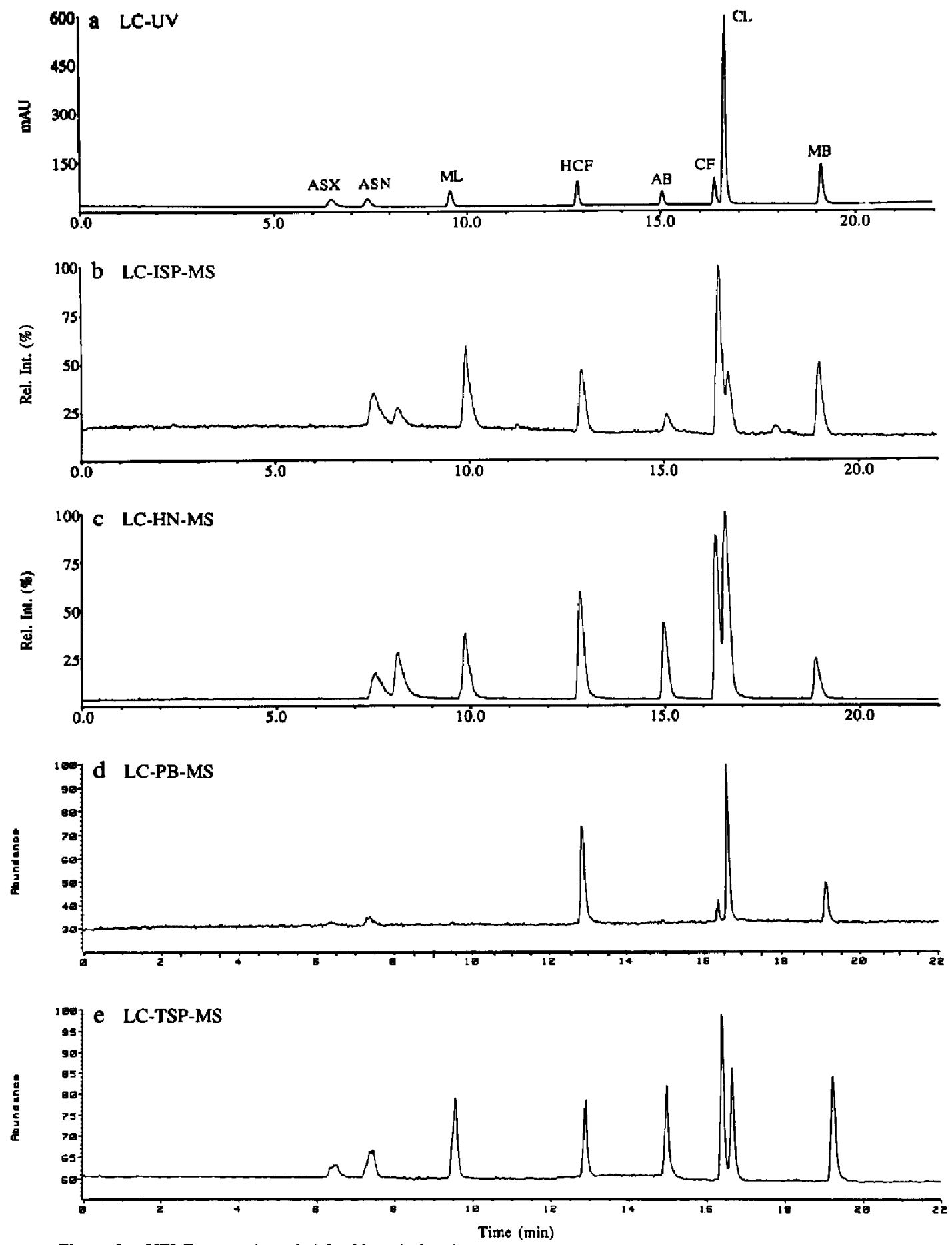

Figure 3. HPLC separation of eight $N$-methyl carbamate pesticides with detection by (a) UV (214 nm) and mass spectrometry using (b) ISP. (c) APCI, and (d) PB-EI, and (e) TSP interfaces. Conditions: column, $250 \times 4.6 \mathrm{~mm}$ i.d. Zorbax RX-C $\mathrm{g} ;$ mobile phase, aqueous methanol for a and c, and modified with formic acid for $b$, all at a flow rate of $1 \mathrm{~mL} / \mathrm{min}$; gradient profile, held at $20 \%$ methanol for $4 \mathrm{~min}$, followed by a linear gradient to $70 \%$ methanol in 11 min and held for 5 min; split ratios, 1:20 for $b$ and 3:2 for $\mathrm{d}$, postcolumn addition of $0.2 \mathrm{~mL} / \mathrm{min} 0.5 \mathrm{M}$ ammonium acetate for e; injection volume, $20 \mu \mathrm{L}\left(20 \mu \mathrm{g} / \mathrm{mL}\right.$ for a and $d_{s}$ and $2 \mu \mathrm{g} / \mathrm{mL}$ for $\mathrm{b}, \mathrm{c}$, and e). 
the two HP1090 liquid chromatographs used in this investigation (see Experimental), and the time scales have been adjusted in order to make comparisons between the traces easier.

LC/ISP / MS. Although the ISP interface can handle flow rates of up to $200 \mu \mathrm{L} / \mathrm{min}$ using full gradient elution, a flow rate of $50 \mu \mathrm{L} / \mathrm{min}$ has been found in this laboratory to be the optimal for routine LC/MS applications. The 4.6- $\mathrm{mm}$ i.d. column was therefore coupled to the ISP interface via a split arrangement (see Experimental) adjusted such that only $50 \mu \mathrm{L} / \mathrm{min}$ was delivered to the mass spectrometer. The SIM LC/ISP/MS reconstructed in chromatogram (RTC) comprising the eight $\mathrm{MH}^{+}$ions, and generated from a $20-\mu \mathrm{L}$ injection of a $2 \mu \mathrm{g} / \mathrm{mL}$ mixture, is presented in Figure $3 \mathrm{~b}$. There is good correspondence between the UV and RIC traces, and no significant loss in chromatographic performance is observed with the split arrangement. The addition of formic acid to the mobile phase does not appear to affect the separation. This close correspondence allows the rapid confirmation of individual chromatographic peaks by mass as well as by retention time. With $40 \mathrm{ng}$ of the individual components injected on-column and a split ratio of 20:1, each peak actually represents only 2 ng reaching the ISP interface.

To improve the selectivity of the LC/ISP/MS method, the instrument was configured for MS/MS using SRM, and the $20-\mu \mathrm{g} / \mathrm{mL}$ mixture of eight compounds was reanalyzed. The LC/ISP/MS/MS reconstructed ion chromatogram, comprising the CID reactions of the $\mathrm{MH}^{+}$ions to the $[\mathrm{MH}-57]^{+}$fragment for ML, CL, MB, CF, and HCF, and to the [MH-75] ${ }^{+}$ fragment for $A B, A S X$, and $A S N$ is shown in Figure 4a. The chromatogram is almost identical to the LC/MS trace in Figure $3 b_{r}$ except that the individual responses now reflect the relative abundances of the most intense fragment ions in the MS/MS spectra characterized by FIA (Table 2). The anticipated improvements in selectivity for complex real-world samples are obtained at the expense of sensitivity; for the carbamate standards the SRM response was typically an order of magnitude less than that obtained in the corresponding SIM analysis.

A different MS/MS scan mode that can be utilized with the triple quadrupole, not only for additional selectivity in target compound analysis but also for the detection of related compounds, is the neutral loss scan. In this mode of operation, $Q_{1}$ and $Q_{3}$ are scanned together with a fixed mass difference to monitor some characteristic loss of a neutral species. For the carbamates, an obvious example is the loss of the neutral methyl isocyanate moiety. The instrument was reconfigured to monitor the loss of $57 \mathrm{u}$, with Q1 scanning from 150 to 250 , and the standard mixture was reinjected. The resultant total ion current (TIC) trace is presented in Figure $4 \mathrm{~b}$. As could be predicted from examination of the ISP/MS/MS spectra in Table
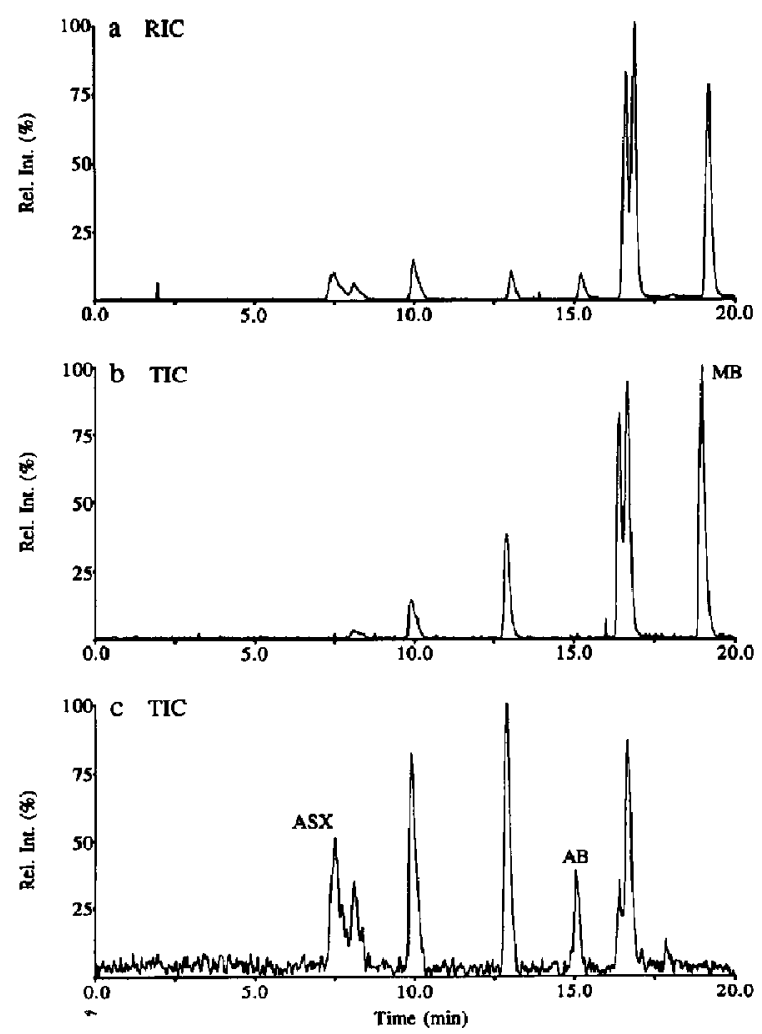

Figure 4. Analysis of carbamate pesticides by LC-ISP/MS/MS using different scan modes. (a) SRM RIC comprising the dissociations of the protonated molecules to the $[\mathrm{MH}-57]^{+}$fragment ions of $M L, C L, M B, C F$, and $H C F$, and to the $[\mathrm{MH}-75]^{+}$ fragment for $A B, A S X$, and ASN. ( $b$ and $c$ ): Neutral loss TIC $(150-250 \mathrm{u})$ traces for the losses of 57 and $75 \mathrm{u}$, respectivcly. $L C$ conditions as in Figure $3 \mathbf{b}$, except $20 \mu \mathrm{g} / \mathrm{mL}$ solution used. MS/MS conditions as in Figure 1c.

2, no response was obtained for $A B$ and $A S X$, which do not undergo this transition. It is interesting to note that the response for HCF is composed of the loss of 57 u from both the protonated molecule $(m / z$ 238) and the fragment ion at $m / z 220$ which appears in the conventional ISP spectrum (described earlier). Figure $4 \mathrm{c}$ presents the LC/MS/MS TIC trace obtained for the related neutral loss of $75 \mathrm{u}$ (carbamic acid), and it can be seen that both $A B$ and ASX now give a response although MB no longer appears. These neutral loss scan modes, used in combination, offer the potential of screening suspect extracts for putative carbamates. In contrast, the SRM approach offers high selectivity for the determination of target analytes.

$L C / A P C I / M S$. The HN interface was connected directly to the $4.6-\mathrm{mm}$ i.d. column operating at 1 $\mathrm{mL} / \mathrm{min}$. Monitoring the $\mathrm{MH}^{+}$ions of $\mathrm{ASN}, \mathrm{MB}$, and $\mathrm{CF}$, the $[\mathrm{MH}-57]^{+}$ions of $\mathrm{ML}$ and $\mathrm{CL}$, and the [MH$75]^{+}$ions of AB, ASX, and HCF (see Table 2), the corresponding LC/APCI/MS RIC obtained from a 20- 
$\mu \mathrm{L}$ injection of the $2 \mu \mathrm{g} / \mathrm{mL}$ test mixture of eight carbamates is shown in Figure $3 \mathrm{c}$. The chromatographic integrity is again maintained, and the interface provides an improved response for all of the components over that observed for ISP, with the largest signals provided by CF and CL. During the gradient elution profile no attempt was made to vary the $\mathrm{HN}$ interface temperature, which was maintained at $300{ }^{\circ} \mathrm{C}$, the temperature used in the preliminary FIA experiments. LC/MS/MS methodologies analogous to those described above for ISP were also applied under APCI conditions with similar success (data not shown). The slight increase in chromatographic peak width observed in the API traces (Figure $3 b$ and c) are due to the dead volume introduced by the presence of the postcolumn injector used in these particular systems. In future work, a precolumn switching valve will be employed for FIA.

LC/PB/MS. The LC/PB/MS SIM RIC obtained with the PB interface operating in the EI mode is presented in Figure $3 \mathrm{~d}$. The RIC is composed of the major fragment ions observed in the spectra of the individual carbamates (see Table 2). Since the optimal flow rate for the $\mathrm{PB}$ interface is $\sim 400 \mu \mathrm{L} / \mathrm{min}$, this required a split ratio of $3: 2$. While the chromatographic integrity is equally maintained with the $\mathrm{PB}$ interface, the results were not encouraging with regard to the sensitivity of the techrique. For example, it should be noted that the RIC in Figure 3d was obtained by using the same solution as that used in the UV study, i.e., 10 times more concentraled than that used for the other three LC/MS interfaces. Each peak therefore represents $400 \mathrm{ng}$ injected, or $160 \mathrm{ng}$ delivered to the spectrometer based on the split ratio. In addition, not all of the carbamates were detected; no response was observed for $A B$ by monitoring either the [M-75] ${ }^{+'}$ ion at $m / z 115$ or the additional fragment ion at $m / z 89$. The responses for ML $(m / z 105)$ and ASX $(m / z 68)$ are also notably weak. Although improved responses were obtained for $A B, A S X$, and ASN using methane $C I$, this mode of ionization was generally less sensitive than EI and also, for reasons unknown at present, gave rise to significant chromatographic peak broadening for all of the pesticides.

The use of ammonium acetate to enhance positive ion abundances in LC/PB/MS has recently been reported [32], and further experiments were undertaken in order to determine whether this "carrier process" could provide additional sensitivity for the carbamate pesticides. For CL (100 $\mathrm{ng}$ injected), an enhancement factor of 1.55 under EI conditions was obtained with the addition of $0.01 \%$ ammonium acetate to the mobile phase, which is in excellent agreement with the value of 1.6 reported previously for this compound [32]. However, the effect was not the same for all of the pesticides and for some, the addition of ammonium acetate was in fact detrimental. Under CI conditions, at the $500 \mathrm{ng}$ level, the mean enhancement factor for the other carbamates were; $\mathrm{CL}(1.6)$, ML (0.7), AB (0.2), ASX (2.9), ASN (2.6), MB (1.0), CF (2.6), and HCF (1.2). Additional experiments also revealed that the enhancement effect was dependent on the concentration of the analyte. For an injection of $100 \mathrm{ng}$ of $\mathrm{CL}$, again under $\mathrm{CI}$ conditions, an enhancement factor of 4.8 was observed with the addition of ammonium acetate.

LC / TSP / MS. In contrast to the results obtained with the PB interface, the TSP results are more comparable with those obtained by the API techniques. As with the HN interface, the $4.6-\mathrm{mm}$ column could be coupled directly to the TSP interface. With the postcolumn addition of ammonium acetate, the total flow rate was $1.2 \mathrm{~mL} / \mathrm{min}$. As mentioned above in the summary of the FIA studies, the response of the carbamates is dependent on the TSP vaporizer temperature, which is itself dictated by the proportion of methanol in the mobile phase. During the mobile phase gradient the vaporizer temperature was therefore ramped from 110 to $102{ }^{\circ} \mathrm{C}$ in parallel with the increasing percentage of methanol. The corresponding LC/TSP/MS RIC is shown in Figure 3e. Comprised of the $\left[\mathrm{M}+\mathrm{NH}_{4}\right]^{+}$ions of all of the carbamates, the RIC is very similar to those obtained by the API techniques. Additional experiments were conducted in which the concentration of ammonium acetate was varied by changing the flow rate of the 0.5 $M$ stock solution introduced postcolumn. At 0.3 $\mathrm{mL} / \mathrm{min}(0.12 \mathrm{M})$ a lower SIM LC/MS response was observed for all of the carbamates except HCF which showed a 1.2-fold increase. At $0.1 \mathrm{~mL} / \mathrm{min}(0.05 \mathrm{M})$, $\mathrm{ML}, \mathrm{AB}, \mathrm{CF}$, and $\mathrm{CL}$ all showed an increase in response, while that for MB and HCF were lower. The sulfonated carbamates, showing at optimum response at $0.2 \mathrm{~mL} / \mathrm{min}$, were the least sensitive to the ammonium acetate concentration. The most sensitive was $\mathrm{CL}$, showing an almost twofold increase from 0.12 to $0.05 \mathrm{M}$. The effect also appeared to be dependent on the concentration of the analyte, although this was not investigated further.

At the level used to generate the RICs presented in Figure $3 b$ and $c$ ( $40 \mathrm{ng}$ each injected), it was possible to obtain full-scan LC/MS spectra for all of the carbamates using ISP and APCI. The LC/MS spectra were essentially identical to those obtained by FLA described above for both interfaces, although notably of poorer quality for LC/ISP/MS. Unfortunately, even though $400 \mathrm{ng}$ of each component was injected for $\mathrm{LC} / \mathrm{PB} / \mathrm{MS}$, only $\mathrm{CL}, \mathrm{MB}$, and HCF provided full-scan LC/MS spectra of even partial resemblance to those obtained by FIA.

To more clearly demonstrate the relative sensitivities of the different LC/MS approaches a representative carbamate, $C L$, was analyzed separately by LC/MS using each of the four interfaces. Appropriate dilutions of the $\mathrm{CL}$ stock solution were made in each case to provide SIM LC/MS traces of similar quality 
with regard to signal to noise. The results of this study are presented in Figure 5. In order of increasing sensitivity, the amounts of $\mathrm{CL}$ injected to produce these traces were; PB-EI (25 ng), TSP (2 ng), ISP (2 $\mathrm{ng})$, and APCI (100 pg). It is important to remember, however, that with the postcolumn split used in $\mathrm{PB}$ and ISP the actual amounts of CL reaching the mass spectrometer in these techniques represents only 8.3 ng and $100 \mathrm{pg}$, respectively. A similar sensitivity order among the interfaces was found for most of the other carbamates, although TSP provided slightly better detection limits than ISP for $A B$ and ASN. The SIM LC/MS method detection limits of all the carbamates examined by the four techniques are tabulated in Table 2. The values obtained for APCI compare favorably with those currently available by HPLCFLD. While several carbamates, other than $\mathrm{CL}$, were also analyzed individually by using all four interfaces, the majority of the detection limits (Table 2) were estimated from the relative responses obtained in the LC/MS analyses of the standard mixtures. In Table 3, the detection limits of $C L, C F$, and $A B$, obtained with the various interfaces in this investigation are also compared with those reported previously in the literature. It can be seen that APCI using the HN interface represents a significant advance in terms of sensitivity over the earlier LC/MS approaches.

While the primary focus of this work was the evaluation of LC/API/MS as a confirmatory spectroscopic method, it is felt that the eventual aim of any LC/MS approach should be its application to routine, quantitative analyses with high sample throughout (i.e., cost effective). During this phase of the investigation, the linearity and reproducibility of the various
Figure 5. Analysis of carbaryl (CL) by (a) LC-PB/MS (25 ng), (b) LC-TSP/MS (2 ng), (c) LC-ISP/MS (2 ng), and (d) LC-APCI-MS (100 pg). Conditions identical to those used in Figure 3. Values given represent amount injected on-column.
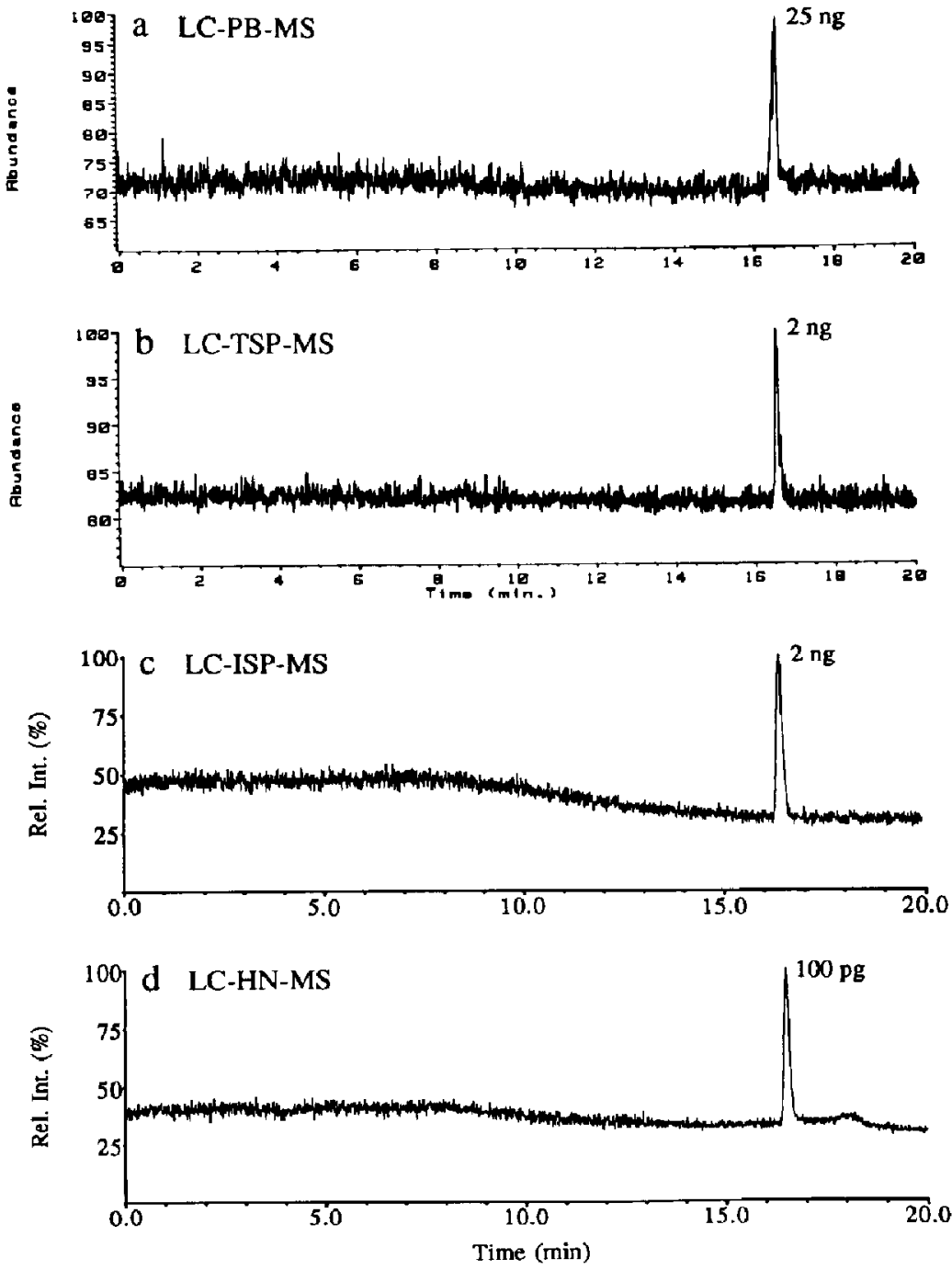


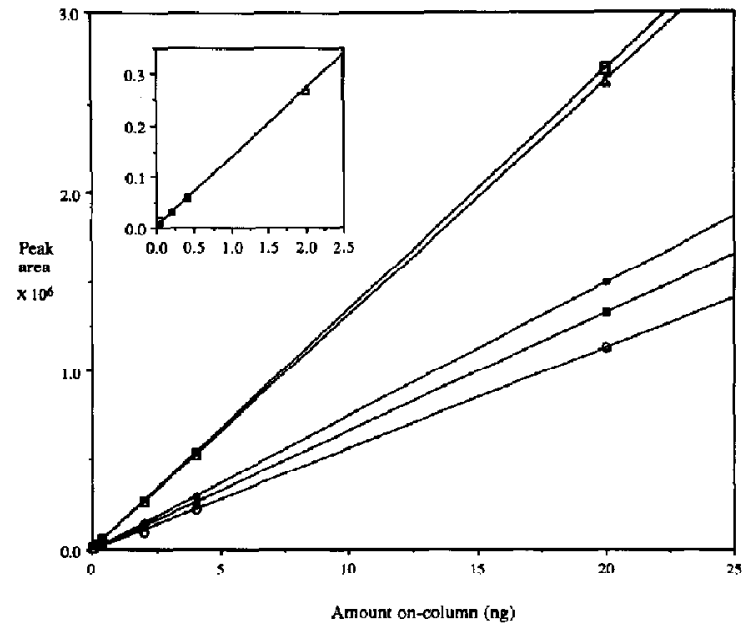

Figure 6. LC-APCl/MS standard curves generated from duplicate $20-\mu \mathrm{L}$ injections of $\mathrm{ML}(\bullet), \mathrm{AB}(O), \mathrm{CL}(\square), \mathrm{MB}(\square)$, and $\mathrm{HCF}(\triangle)$. Inset shows expanded region for $\mathrm{CL}$.

LC/MS systems were also examined in this context. Again the results obtained by APCI stand out from those of the other techniques. Standard APCI calibration curves for five of the carbamates, from duplicate $20 \mu \mathrm{L}$ injections over the range $2-1000 \mathrm{ng} / \mathrm{mL}$, are presented in Figure 6. The slopes of the curves for individual carbamates reflect the relative LC/MS sensitivities given in Table 2 . The inset shows the expanded low level region of the curve for CL. These and other data were typically generated by the LC/APCI/MS system in unattended operation over the course of a working day, with an autosampler providing the start signal for the mass spectrometry data system. Although the reproducibility provided with TSP and ISP was not as good as that obtained by $A P C I$, the former technique provided a slightly wider linear dynamic range $(0.05-20 \mu \mathrm{g} / \mathrm{mL})$ than both the API techniques. The quantitative aspects of LC/API/MS will be considered further in future publications. Although the PB interface provided a linear response over a limited range, the curve had a nonzero (positive) intercept on the ordinate axis (amount injected) and an increasingly nonlinear response (convex downwards) at low concentrations. This feature of the PB interface has been observed in this labora- tory with other compound classes such as the tetracycline antibiotics.

One aspect of this work which should be considered more closely is the split arrangement used for both PB and ISP. Being pressure controlled, it is possible that there will be some variability in the split-ratio during a gradient elution profile. This could have an effect on the day-to-day reproducibility of the techniques and would require the use of a balanced split or an appropriate internal standard. The use of 2.1- and 1.0-mm i.d. microbore columns for PB and ISP, respectively would remove the need for the postcolumn split, although at the expense of column capacity; an important factor in trace analysis (see below). Unfortunately, the particular base-deactivated stationary phase used in this investigation is not available in microbore format, nor available in bulk packing such that the $2.1-$ and $1.0-\mathrm{mm}$ i.d. columns could be packed in-house.

\section{Determination of $M L, A B$, and $C L$ in Pepper Extracts}

To more fully evaluate the performance of the LC/MS interfaces for the analysis of these compounds in complex mixtures, three representative carbamates (AB, ML, and CL) were spiked into a homogenate of green peppers at the 0.1-ppm level. The current legal levels in Canada for these pesticides in green peppers are $0.1,0.1$, and 5 ppm, respectively. In parallel with a control homogenate the spiked peppers were extracted (see Experimental), and the extracts subsequently analyzed by all four LC/MS techniques using conditions identical to those described above. In the case of $\mathrm{CL}$, additional confirmatory GC/MS analyses were also undertaken.

None of the three pesticides in the spiked pepper extract could be detected by LC/PB/MS, even using SIM techniques. This is not surprising because the on-column detection limits of the three carbamates on the PB interface (Table 3), even with the addition of ammonium acetate, were all higher than the value of $3.5 \mathrm{ng}$ expected in the spiked sample $(0.175 \mathrm{ng} / \mathrm{mL}$ with $100 \%$ recovery; $20-\mu \mathrm{L}$ injection). The problem is clearly one of analyte transmission through the PB interface because it was possible to confirm the presence of $\mathrm{CL}$ by GC/MS, and all three carbamates by

Table 3. Comparison of detection limits for three representative carbamate pesticides using various LC/MS systems

\begin{tabular}{|c|c|c|c|c|c|c|c|c|c|c|}
\hline \multirow[b]{3}{*}{ Carbamate } & \multicolumn{10}{|c|}{ Detection limit/ng (quantitation ion) } \\
\hline & \multirow[b]{2}{*}{ MW } & \multirow{2}{*}{$\begin{array}{l}\text { Moving } \\
\text { belt }(E I)^{a} \\
\text { SIM }\end{array}$} & \multirow{2}{*}{$\begin{array}{c}\text { DLI } \\
\text { Full-scan } \\
(150-550)\end{array}$} & \multicolumn{2}{|l|}{$\mathrm{TSP}^{\mathrm{c}}$} & \multicolumn{2}{|c|}{ PB-EI } & \multirow[b]{2}{*}{$\begin{array}{l}\text { ISP } \\
\text { SIM }\end{array}$} & \multicolumn{2}{|l|}{ APCI } \\
\hline & & & & $\begin{array}{l}\text { Full-scan } \\
(60-550)\end{array}$ & SIM & $\begin{array}{l}\text { Full-scan } \\
(62-450)^{d}\end{array}$ & SIM & & $\begin{array}{l}\text { Full-scan } \\
(50-350)\end{array}$ & SIM \\
\hline Carboturan (CF) & 221 & $25(165)$ & $50(222)$ & 2 (239) & 0.8 & $240(164)$ & 55 & $0.3(222)$ & $0.5(222)$ & 0.05 \\
\hline Carbaryl (CL) & 201 & $25(145)$ & $40(202)$ & $4(219)$ & 0.8 & $100(144)$ & 10 & $1.0(202)$ & $0.5(145)$ & 0.05 \\
\hline Aldicarb (AB) & 190 & $6(89)$ & $60(191)$ & $4(208)$ & 0.9 & $N A(89)$ & $>500$ & $1.5(191)$ & $1.0(116)$ & 0.07 \\
\hline
\end{tabular}

a From ref 13 . ${ }^{b}$ From ref 15 . ${ }^{c}$ From ref 16 (see also ref 18 ). d From ref 19. 


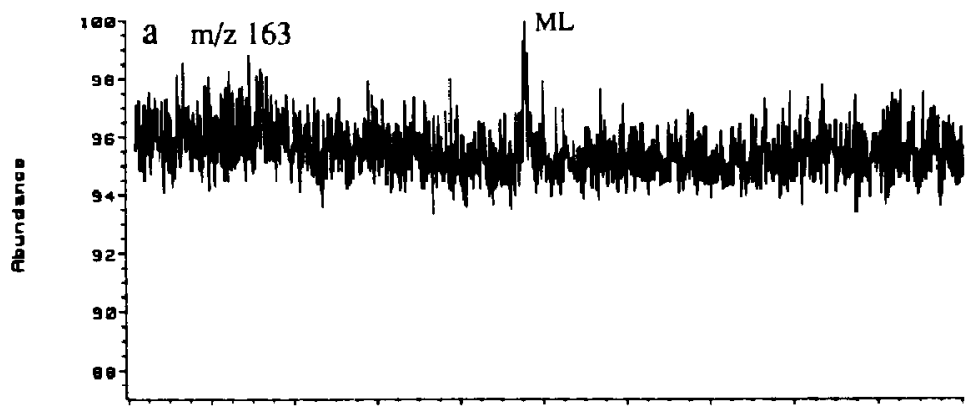

Figure 7. Analysis of spiked (0.1 ppm) green pepper extracts by LC-TSP/MS. SIM traces of (a) the $\mathrm{MH}^{+}$ion of $\mathrm{ML}(m / z 163)$, and the $\left[\mathrm{M}+\mathrm{NH}_{4}\right]^{+}$ions of (b) $\mathrm{AB}$ and (c) CL ( $m / z 208$ and 219 , respectively
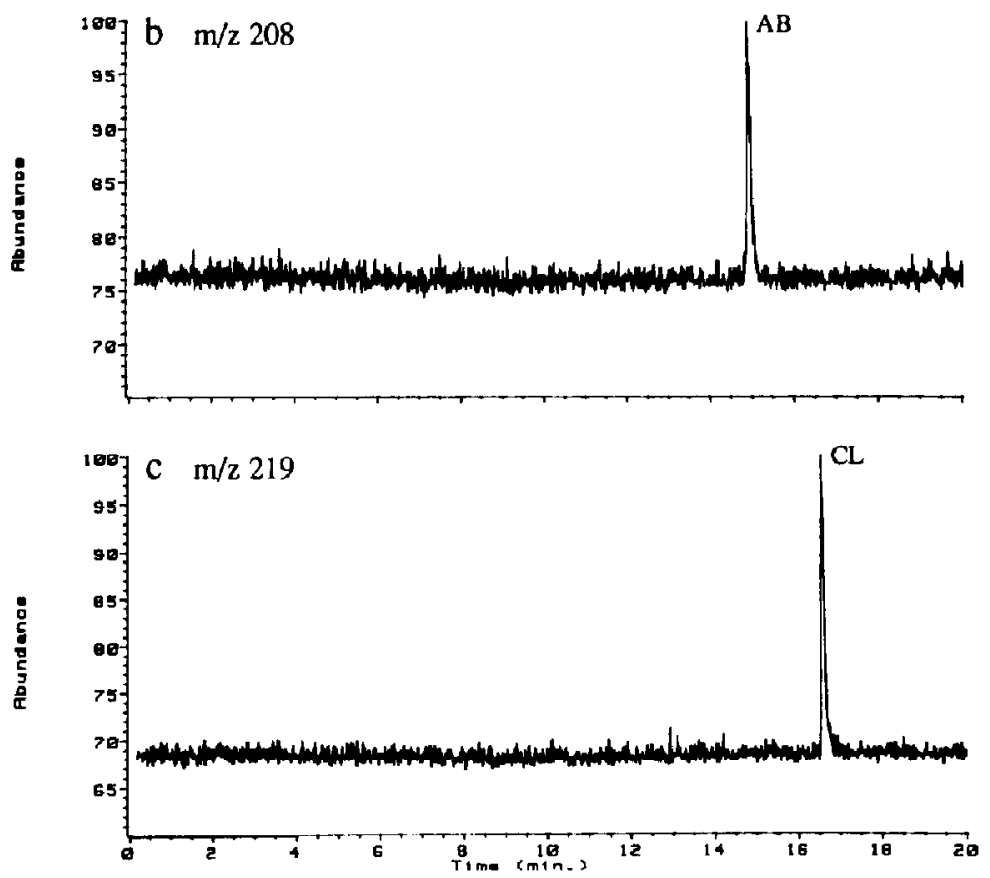

LC/TSP/MS (see below), using the same mass spectrometer as used for the PB work. It is important to note that, during the present investigation, there was no preconcentration of the samples before LC/MS analyses. It should also be possible to inject up to 200 $\mu \mathrm{L}$ on the 4.6-mm i.d. column when using gradient elution without degrading the chromatographic performance. While not within the scope of this comparative exercise, these additional steps, in conjunction with SIM techniques, should provide the sensitivity necessary for confirmatory analyses for the majority of the carbamates examined using the $\mathrm{PB}$ interface. However, for compounds such as $\mathrm{AB}$, even these additional measures may not be sufficient.

As indicated above, it was possible to confirm all three pesticides by TSP, and the SIM chromatograms of the $\mathrm{MH}^{+}$ion of $\mathrm{ML}(m / z 163)$ and the $\left[\mathrm{M}+\mathrm{NH}_{4}\right]^{+}$ ions of $\mathrm{AB}$ and $\mathrm{CL}(\mathrm{m} / \mathrm{z} 208$ and 219 , respectively) are presented in Figure $7 a-c$, respectively. Good responses are observed at the correct retention times for both $A B$ and $C L$, although the response for $M L$ is disappointingly weak. A higher response was obtained for ML by monitoring the $\left[\mathrm{M}+\mathrm{NH}_{4}\right]^{+}$ion at $m / z 180$, although this trace was unfortunately complicated by a broad interference peak (not shown).

All three pesticides were similarly confirmed by LC/ISP/MS using SIM of the individual $\mathrm{MH}^{+}$ions. The appropriate trace for $\mathrm{CL}$ is shown in Figure $8 \mathrm{~b}$. An additional peak is observed in the chromatogram eluting just after $\mathrm{CL}$. This is due to an endogenous compound, as indicated by a corresponding peak in the SIM trace of the control sample (Figure 8a). In order to increase the confidence of the LC/ISP/MS analyses, the selectivity of the method was increased by performing additional LC/ISP/MS/MS experiments, using SRM of the CID reactions for the three $\mathrm{MH}^{+}$ions to their respective major fragment ions (see Table 2). The SRM chromatogram obtained for CL by monitoring the dissociation of the protonated molecule to its $[\mathrm{MH}-57]^{+}$fragment ion $(m / z 202>m / z 145)$ is 

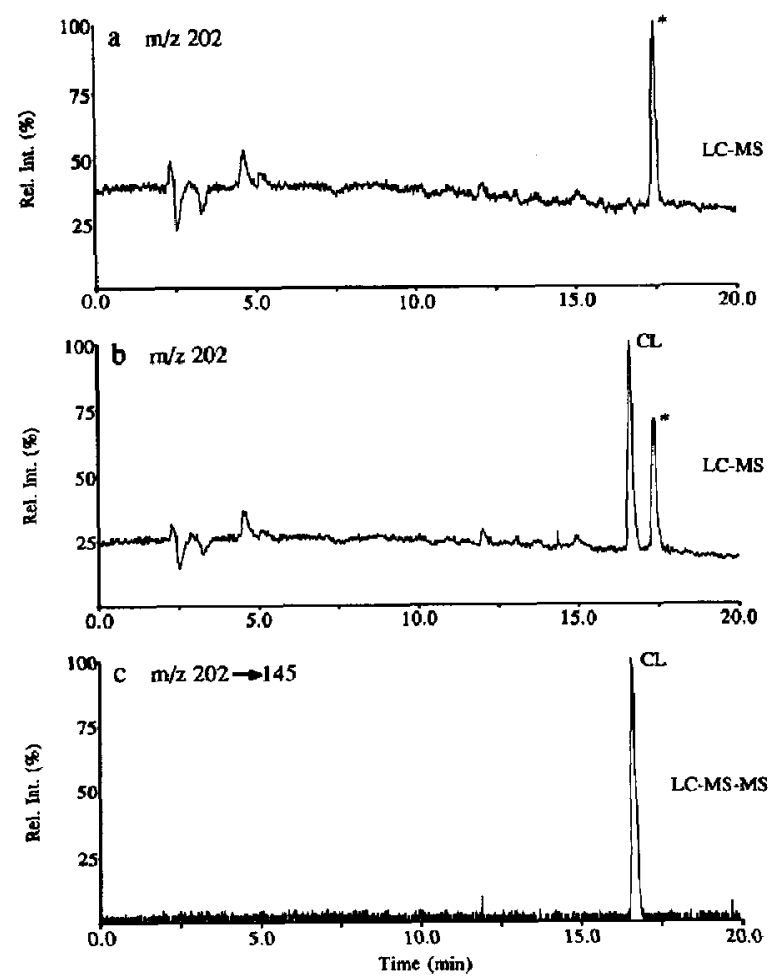

Figure 8. Analysis of green pepper extracts by ( $a$ and $b$ ) LC-ISP/MS and (c) LC-ISP/MS/MS. SM traces of the protonated molecule of CL ( $m / z$ 202) from (a) control and (b) spiked $(0.1 \mathrm{ppm})$ pepper extracts; (c) SRM trace of dissociation of $\mathrm{MH}^{+}$ ion of $\mathrm{CL}(m / z 202)$ to its $[\mathrm{MH}-57]^{+}$ion $(m / z$ 145) from spiked pepper extract. *Endogenous peak observed in control extract.

shown in Figure 8c. The increased selectivity is immediately apparent from the reduction in chemical noise and the absence of the interfering endogenous peak. While ML was similarly confirmed by SRM using the same loss of $57 u(m / z 163>106)$, it was not possible to obtain a response for $\mathrm{AB}$ by monitoring the $\mathrm{CID}$ reaction of the $\mathrm{MH}^{+}$ion $(m / z$ 191) to its major [MH$751^{+}$fragment ion $(\mathrm{m} / z$ 116) owing to the abundance of the precursor ion. It was, however, possible to obtain an adequate signal monitoring the reaction $m / z 116>89$.

In comparison with the other techniques, the results obtained by LC/APCI/MS were impressive. Not only was it possible to detect all three carbamates using SIM of both their major fragment and $\mathrm{MH}^{+}$ ions, but the technique also provided confirmatory full-scan spectra of each of the individual analytes. The chromatograms corresponding to the $[\mathrm{MH}-57]^{+}$ ions of $M L(m / z 106)$ and $C L(m / z 145)$ and the [MH-75] $^{+}$ion of $\mathrm{AB}(m / z$ 116), extracted from the full-scan (50-350 u) TIC, are presented in Figure $9 a, c$, and $b$, respectively. The quality of these chromatograms is comparable to that provided by SIM using either TSP or ISP. The background-subtracted,
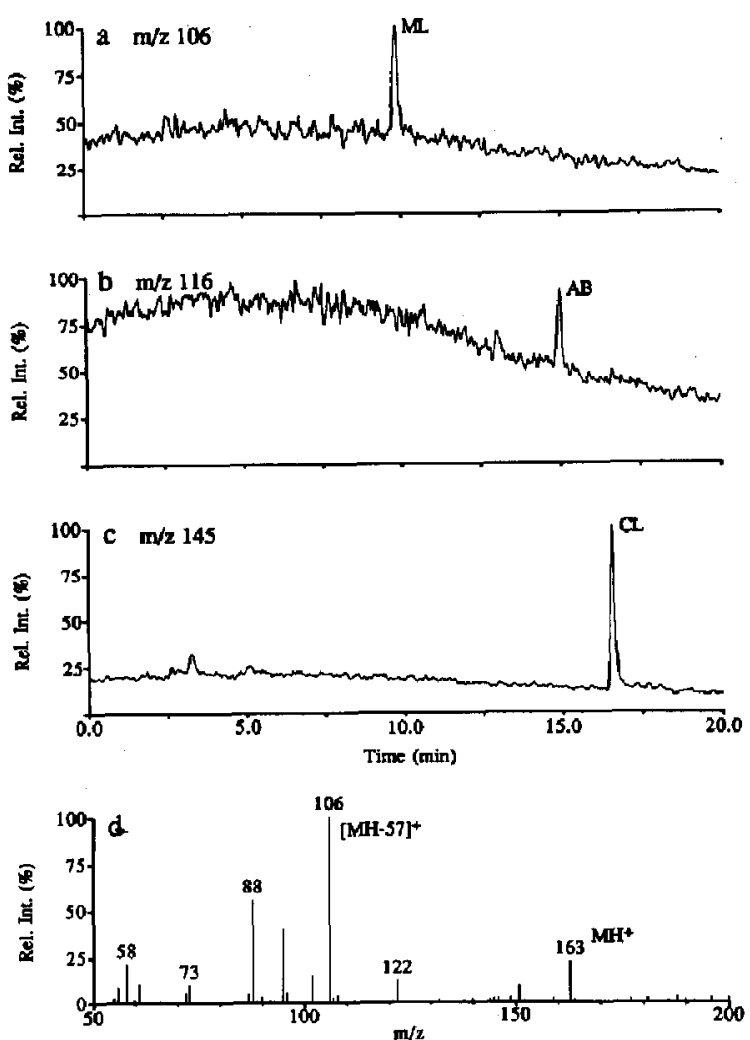

Figure 9. Analysis of spiked (0.1 PPm) green pepper extract by LC-APCI/MS. Mass chromalograms of (b) the [MIH-75] ${ }^{+}$ion of $\mathrm{AB}\left(\mathrm{m} / z\right.$ 116) and the $[\mathrm{MH}-57]^{+}$ions of (a) $\mathrm{ML}$ and (c) $\mathrm{CL}(\mathrm{m} / z$ 106 and 145, respectively), extracted from full-scan (50-350 u) TIC. (d) background-subtracted spectrum of ML taken from top of peak in $\mathbf{a}$.

full-scan spectrum of ML taken from the peak in Figure $9 \mathrm{a}$ is presented in $9 \mathrm{~d}$, and compares favorably with that of the pure compound obtained by FIA (Figure 1d). No major coeluting interferences were detected in the full-scan APCI analysis. If additional confirmatory evidence should ever be required, LC/MS/MS methodologies similar to those described above for ISP could also be applied in the case of APCI.

Comparison of the LC/MS SIM peak areas from a standard solution of the three pesticides with those from the analysis of control peppers spiked after extraction revealed no significant losses of analyte signal due to interferences or matrix effects. Recoveries for the spiked carbamates were then estimated from comparisons of the LC/MS SIM peak areas with those obtained for known injections of the standard solutions. These semiquantitative results (single point calibration assumed valid, single injection in each case) are presented in Table 4, which also includes GC/MS data for $\mathrm{CL}$ for comparison. Although the ISP recoveries appear to be consistently higher than those esti- 
Table 4. Estimated recoveries from the various LC/MS techniques

\begin{tabular}{lcccc}
\hline & \multicolumn{3}{c}{ Estimated recoveries (\%) } \\
\cline { 2 - 4 } Pesticide & LC-MS & \\
\cline { 2 - 4 } & TSP & ISP & APCl & GC/MS \\
\hline \hline ML & 87 & 93 & 85 & 105 \\
AB & 84 & 88 & 79 & NA \\
CL & 92 & 106 & 86 & 102 \\
\hline
\end{tabular}

All values are single SIM measurements; based on a single-point calibration.

mated by TSP and APCI, there is an acceptable degree of correlation between these LC/MS results, which are obtained on two different instruments using three very different ionization techniques.

\section{Conclusions}

The purpose of the present work was to evaluate API techniques for the analysis of $N$-methyl pesticides by LC/MS, through comparison with more established LC/MS interfaces. Unfortunately, it was not possible to perform these comparisons using the same mass spectrometer in all cases. However, since both the single and triple quadrupole systems used in this investigation are of comparable rod dimensions and are of modern design, it is believed that the differences observed reflect differences in the LC/MS interfaces and not the inherent sensitivity of the particular spectrometers.

Of the two LC/MS interfaces which do not involve API, the TSP interface performed well, providing SIM detection limits of a few nanograms. The principal drawback of the TSP interface was found to be its well-documented problem of fluctuating ion signals $[18,20]$. Nonetheless, TSP offers a viable technique for both confirmatory analyses of carbamate pesticides at the regulatory level ( $\sim 0.1 \mathrm{ppm})$, and quantitative analyses provided that a suitable internal standard is used. In contrast, the PB interface (in our hands) did not perform well for the carbamates. The interface, which was used on the same mass spectrometer as used for the TSP study, appeared extremely ineffcient in transporting these analytes into the ion source. This is indicated by the poor sensitivity (Table 2) and the nonzero intercept of the calibration curves. Although the results obtained with the PB interface were disappointing, further improvements in interface design and the use of high energy dynode electron multipliers should provide the additional sensitivity required for confirmatory analyses. However, the problem of nonlinearity with the PB interface still needs to be addressed if it is to be used for quantitative analyses.

Each of the API interfaces performed well in this investigation, with the heated nebulizer interface utilizing APCI having a clear advantage over the other techniques with respect to sensitivity (Tables 2 and 3), linearity (Figure 6), and breadth of applicability to all the carbamates studied. APCI provides both protonated molecules for molecular weight information and abundant fragment ions for structural confirmation. The ISP interface performed on comparable terms with the TSP for this class of compound. It is thought that the ISP technique will bc morc appropriate for even more thermally labile and polar analytes, particularly as, increasingly, the compounds of environmental concern tend to higher molecular weight and increased polarity. Indeed, it is the range of analytes covered by these two readily interchangeable interfaces that makes the API system so attractive.

One other important feature of both the API techniques is their user friendliness and analytical ruggedness compared to other LC/MS interfaces. The fact that the high vacuum is not physically connected with the chromatographic system is very forgiving to the inexperienced or novice spectroscopist. The ability to change or repair interfaces and even clean the source while in operation is also a major benefit to the analyst.

Encouraged by the results of this preliminary investigation, we are currently extending our studies to representatives from a wider variety of pesticide classes and will be placing more emphasis on the quantitative aspects of LC/API/MS.

\section{Acknowledgments}

The authors are pleased to acknowledge the help of Dr. R. K. Boyd in the preparation of this manuscript.

\section{References}

1. Engelhardt, H.; Lillig, B. Chromatographia 1986, 21, 136-142.

2. Hall, R. C.; Harris, D. E. J. Chromatogr. 1979, 169, 245-259.

3. Trehy, M. L.; Yost, R. A.; Dorsey, J. G. Anal. Chem. 1986, $58,14-19$.

4. Coburn, J. A.; Ripley, B. D.; Chau, A. S. Y. J. Assoc, Off. Anal. Chem. 1976, 59, 188-196.

5. Sparacino, C. M.; Hines, J. W. J. Chromatogr. Sci. 1976, 14, $549-556$.

6. Krause, R. T. J. Chrumutuyr. Sci. 1988, 442, 333-343.

7. Moye, H. A.; Scherer, S. J.; St. John, P. A. Anal. Lett. 1977, $10,1049-1073$.

8. Krause, R. T. J. Chromatogr. Sri. 1978, 16, 281-288

9. Hill, K. M.; Hollowell, R. H.; Dal Cortivo, L. A. Anal. Chem. 1984, 56, 2465-2468.

10. Krause, R. T.; August, E. M. I. Assoc. Off. Anal. Chem. $1983,66,234-240$.

11. Krause, R. T. J. Assoc. Off. Anal. Chem. 1980, 63, 1114-1124.

12. Games, D. E. In Applications of Mass Spectrometry in Food Science; Gilbert, J., Ed.; Elsevier: New York, 1987; pp 193-223.

13. Wright, L. H. J. Chromatogr. Sci. 1982, 20, 1-6.

14. Cairns, T.; Siegmund, E. G.; Doose, G. M. Biomed. Mass Spectrom. 1983, 10, 24-29.

15. Voyksner, R. D.; Bursey, J. T. Anal, Chem, 1984, 56, 1582-1587.

16. Voyksner, R. D.; Bursey, J. T.; Pellizzari, E. D. Anal. Chem. 1984, 56, $1507-1514$. 
17. Hammond, I.; Moore, K.; James, H.; Watts, C. J. Chromatogr. 1989, 474, 175-180.

18. Bellar, T. A.; Budde, W. L. Amal. Chem. 1988, 60, 2076-2083.

19. Behymer, T. D.; Bellar, T. A.; Budde, W. L. Anal. Chem. 1990, 62, 1686-1690.

20. Chiu, K. S.; Van Langenhove, A.; Tanaka, C. Biomed. Enviran. Mass Spectrom. 1989, 18, 200-206.

21. Kalinoski, H. T.; Wright, B. W.; Smith, R. D. Biomed. Environ. Mass Spectrom. 1986, 13, 33-45.

22. Berry, A. J.; Games, D. E.; Mylchreest, I. C.; Perkins, J. R.; Pleasance, S. Biomed. Environ. Mass Spectrom. 1988, 15, 105-109.

23. Whitehouse, C. M.; Dreyer, R. M.; Yamashita, M.; Fenn, J. B. Anal. Chem. 1985, 57, 675-679.

24. Huang, E. C.; Wachs, T.; Conboy, J. J.; Henion, J. D. Anal.

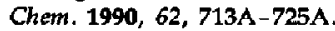

25. Bruins, A. P.; Covey, T. R.; Henion, J. D. Anal. Chem. 1987, $59,2642-2646$.
26. Pleasance, S.; Quilliam, M. A.; deFreitas, A. S. W.; Marr, J. C.; Cembella, A. D. Rapid Commun. Mass Spectrom. 1990, 4, 206-213.

27. Anacleto, J. F.; Ramaley, L.; Boyd, R. K.; Pleasance, S.; Quilliam, M. A.; Sim, P. G.; Benoit, F. M. Rapid Commun. Muss Spectrum. 1991, 5, 149-155.

28. Analytical Procedures for Pesticide Residues in Foods; Health Protection Branch, National Health and Welfare Canada, 1986.

29. Holmstead, R. L.; Casida, J. E. I. Assoc. Off. Anal. Chem. 1975, 58, 541-547.

30. Trehy, M. L.; Yost, R. A.; McCreary, J. J. Anal. Chem. 1984, $56,1281-1285$.

31. Hummel, S. V.; Yost, R. A. Org. Mass. Spectrom. 1986, 21, 785-791.

32. Bellar, T. A.; Behymer, T. D.; Budde, W. L. J. Am. Soc. Mass Spectrom. 1990, 1, 92-98. 